\title{
Efeitos da Hipertensão Arterial Induzida sobre a Complacência e Pressão de Perfusão Encefálica em Hipertensão Intracraniana Experimental: Comparação entre Lesão Encefálica Criogênica e Balão Şubdural *
}

\section{Effects of Induced Hypertension on Brain Compliance and Perfusion Pressure in Experimental Intracranial Hypertension: Comparison between Cryogenic Brain Injury and Subdural Balloon}

Nelson Mizumoto, TSA ${ }^{1}$; Humberto Katsuji Tango, TSA ${ }^{2}$; Marcelo Lacava Pagnocca, TSA ${ }^{3}$

\section{RESUMO}

Mizumoto N, Tango HK, Pagnocca ML - Efeitos da Hipertensão Arterial Induzida sobre a Complacência e Pressão de Perfusão Encefálica em Hipertensão Intracraniana Experimental: Comparação entre Lesão Encefálica Criogênica e Balão Subdural

JUSTIFICATIVA E OBJETIVOS: O trauma cranioencefálico (TCE) pode elevar a pressão intracraniana (PIC) e reduzir a complacência encefálica (CE). Diferentes lesões são aplicadas em modelos de TCE que estudam as mesmas variáveis. Como são usadas indistintamente, o objetivo é comparar a PIC e a CE em dois modelos de TCE.

MÉTODO: Dezoito cães machos, anestesiados, ventilados e distribuídos aleatoriamente em dois grupos: BS - balão subdural $(n=9)$ e $L C$ - lesão criogênica $(n=9)$. Análise da PIC, CE e pressão de perfusão encefálica (PPE) em cinco momentos: fim da preparação (M0), encéfalo normal (M1), início da lesão (M2), término da lesão (M3) e lesão estabelecida (M4). CE é a variação da PIC durante hipertensão arterial induzida (HAl) em $50 \mathrm{mmHg}$ em M1 e M4. PPE = pressão arterial média (PAM) - PIC. Teste $t$ de Student pareado para o mesmo grupo em momentos diferentes e $t$ de Student para duas amostras distintas para o mesmo momento entre os grupos.

Resultados: A PAM foi semelhante nos grupos nos momentos estudados ( $p=$ 0,31 em M0; $p=0,25$ em M1; $p=0,31$ em M2; $p=0,19$ em M3; $p=0,05$ em M4). A $P I C$ foi semelhante nos grupos em M0 $(p=0,27)$ e $M 1(p=0,21)$, mas diferente em $M 2(p<0,001)$. APIC tornou-se semelhante nos grupos em $M 3(p=0,39)$ e $M 4(p=$ $0,98)$, elevou-se no BS em M1 $(p=0,04)$ e M2 $(p=0,01)$, mas não em $M 3(p=0,36)$ nem M4 $(p=0,12)$. No LC a PIC aumentou em M1 $(p<0,01)$, M3 $(p<0,001)$ e M4 ( $p$

\footnotetext{
* Recebido do (Received from) Laboratório de Investigação Médica em Anestesiologia Experimental - LIM-08 Disciplina de Anestesiologia do Departamento de Cirurgia da Faculdade de Medicina da Universidade de São Paulo, SP. Apoio da FAPESP - Fundação de Amparo à Pesquisa do Estado de São Paulo (Processo no 99/02663-8)

1. Supervisor em Neuroanestesia do Instituto de Psiquiatria do HCFMUSP. Anestesiologista do Hospital Israelita Albert Einstein, Co-responsável do CET/HCFMUSP, Doutor em Ciências pela Disciplina de Anestesiologia da FMUSP

2. Assistente Colaborador do Instituto de Psiquiatria do HCFMUSP, Instrutor de Ensino do CET-HCFMUSP, Doutorando da Disciplina de Anestesiologia da FMUSP

3. Supervisor em Neuroanestesia da IMSCSP, Co-responsável do CET-IMSCSP. Doutor em Ciências pela Disciplina de Anestesiologia da FMUSP. Supervisor em Neuroanestesia da FMUSP.
}

Apresentado (Submitted) em 23 de agosto de 2004 Aceito (Accepted) para publicação em 17 de janeiro de 2005

Endereço para correspondência (Correspondence to)

Dr. Marcelo Lacava Pagnocca

Rua Piracuama 316/11 Perdizes

05017-040 São Paulo, SP

E-mail: lacava@usp.br

(c) Sociedade Brasileira de Anestesiologia, 2005
$<0,001)$, mas não em M2 $(p=0,18)$. Houve aumento da PPE em $M 1(p<0,001)$ e M4 ( $p<0,001)$, semelhante nos grupos ( $p=0,16$ em M1 e $p=0,21$ em M4). Em M2 houve redução da PPE nos grupos $(p<0,001)$, mais intensa em $L C(p<0,001)$. Em M3 houve aumento da PPE no BS $(p=0,02)$ e redução no $L C(p=0,01)$, o que tornou a PPE semelhante nos grupos $(p=0,43)$. Em M4 houve aumento da PPE semelhante nos grupos $(p=0,16)$.

CONCLUSÕES: O efeito da hipertensão arterial induzida no modelo de LC é comparável ao observado no modelo de BS. Esse tipo de lesão deve ser melhor estudado para estabelecer precisão na proporção entre a sua extensão e a redução da CE, aparentemente, um processo gradual e evolutivo cujos limites ainda não são totalmente conhecidos.

Unitermos: ANIMAIS: cães; SISTEMA NERVOSO CENTRAL: complacência encefálica, hipertensão intracraniana; TRAUMA: cranioencefálico

\section{SUMMARY}

Mizumoto N, Tango HK, Pagnocca ML - Effects of Induced Hypertension on Brain Compliance and Perfusion Pressure in Experimental Intracranial Hypertension: Comparison between Cryogenic Brain Injury and Subdural Balloon

BACKGROUND AND OBJECTIVES: Traumatic brain injury (TBI) may increase intracranial pressure (ICP) and decrease brain compliance (BC). Different injuries are applied to $T B I$ models studying the same variables. Since they are indistinctly used, the objective was to compare ICP and BC in two different TBI models. METHODS: This study involved 18 male dogs anesthetized, ventilated and randomly distributed in two groups: $S B$ - subdural balloon $(n=9)$ and $\mathrm{Cl}$ - cryogenic injury $(n=9)$. ICP, $B C$ and cerebral perfusion pressure $(C P P)$ were evaluated in five moments: end of preparation (M0), normal brain (M1), beginning of injury (M2), end of injury (M3) and established injury (M4). BC is ICP variation during induced hypertension $(\mathrm{IH})$ in $50 \mathrm{mmHg}$ in $\mathrm{M} 1$ and $\mathrm{M} 4$. CPP = Mean Blood Pressure (MBP) ICP. Paired Student's t test was used for the same group in different moments and Student's t test was used for two different samples in the same moment between groups.

RESULTS: MBP was similar for both groups in all studied moments $(p=0.31$ in MO; $p=0.25$ in M1; $p=0.31$ in M2; $p=0.19$ in M3; $p=0.05$ in M4). ICP was similar between groups in $M 0(p=0.27)$ and $M 1(p=0.21)$, however different in M2 ( $p<$ $0.001)$. ICP was similar for both groups in $M 3(p=0.39)$ and $M 4(p=0.98)$, increased for SB in M1 $(p=0.04)$ and M2 $(p=0.01)$, but not in $M 3(p=0.36)$ and M4 $(p=0.12)$. For $C l$, ICP has increased in M1 $(p<0.01), M 3(p<0.001)$ and $M 4(p<$ $0.001)$, but not in M2 ( $p=0.18)$. There has been CPP increase in $M 1(p<0.001)$ and M4 ( $p<0.001)$, with no difference between groups ( $p=0.16$ in $M 1$ and $p=0.21$ in M4). There has been decreased CPP in M2 for both groups $(p<0.001)$, however more severe for $\mathrm{Cl}(p<0.001)$. In M3, there has been increased $C P P$ for $S B(p=$ $0.02)$ and decreased CPP for $\mathrm{Cl}(p=0.01)$, what has made CPP similar for both groups ( $p=0.43)$. CPP has equally increased in M4 for both groups $(p=0.16)$. CONCLUSIONS: Induced hypertension (IH) effect on $\mathrm{Cl}$ model is comparable to what has been observed in the SB model. This type of injury should be better studied to establish precision in the ratio between injury extension and $B C$ decrease, which seems to be a gradual and evolving process, with not totally understood limits.

Key Words: ANIMALS: dogs; CENTRAL NERVOUS SYSTEM: brain compliance, intracranial hypertension, TRAUMA: brain 


\section{INTRODUÇÃO}

O encéfalo é órgão elástico que, em condições normais, pode modificar seu volume discreta e rapidamente pela alteração do conteúdo de líquor e/ou de sangue, eventualmente alterando a pressão intracraniana (PIC), dependendo da complacência encefálica $(C E)^{1,2}$. A complacência do encéfalo - também referida como elastância - pode ser compreendida como medida da viscoelasticidade, ou da rigidez, do tecido nervoso. Em termos matemáticos, elastância representa a variação volumétrica observada em resposta à modificação da pressão de um corpo elástico, enquanto complacência expressa a relação inversa. Em termos biológicos, o conceito exprime capacidade de compensar incremento volumétrico dentro do crânio, particularmente útil em condições patológicas ${ }^{1,3}$. Segundo a doutrina de Monro-Kellie, quando o volume de um dos componentes do encéfalo aumenta, outro(s) deve(m) reduzir-se proporcionalmente, ou a PIC aumenta. A elevação sustentada da PIC acima de $15 \mathrm{mmHg}$ é condição patológica definida como hipertensão intracraniana $(\mathrm{HIC})^{2}$.

O trauma cranioencefálico (TCE), principalmente quando grave, causa $\mathrm{HIC}^{4}$ - originada a partir de água (edema), líquor (hidrocefalia), sangue intravascular (inchaço encefálico ou brain swelling) ou extravascular (hematomas extradural, subdural ou intraparenquimatoso) - e ainda prejudica a autorregulação vascular encefálica (ARVE) ${ }^{5}$, podendo reduzir a pressão de perfusão encefálica (PPE) de modo dependente da pressão arterial média (PAM) ${ }^{6}$. Alem disso, as vítimas de TCE experimentam dor decorrente do trauma e ainda sofrem manipulações diversas durante seu tratamento, como a intubação e aspiração da traquéia, punções cutâneas, sondagem vesical e nasogástrica, estímulos potencialmente nociceptivos que promovem o aumento da PAM. Essa hipertensão induzida pode aumentar o volume de sangue dentro do compartimento intracraniano, especialmente se há redução da ARVE e da CE. Por essas razões o TCE pode alterar a complacência e a perfusão encefálica de modos diversos.

A maneira e a intensidade em que essas alterações ocorrem é dependente do modo, da velocidade e da intensidade em que o TCE promove a HIC, portanto, o modelo experimental é importante determinante dos resultados observados e das conclusões inferidas a partir deles.

Os modelos experimentais conhecidos procuram mimetizar os fenômenos que surgem na agressão ao neurônio decorrentes do TCE. Dois modelos em particular se destacam por apresentar características específicas semelhantes àquelas encontradas nas vítimas de TCE: a redução da complacência e da pressão de perfusão encefálica.

O primeiro desses modelos é obtido com a insuflação gradual de balão localizado no compartimento intracraniano (extradural, subdural ou intraventricular). A principal vantagem consiste em aumentar a PIC de modo controlável ${ }^{7}$, permitindo manter a intensidade em valor e tempo desejados ${ }^{3}$, o que facilita o estudo da complacência e da perfusão encefálica. Contudo, apesar de ser possível atingir valores de HIC que reduzam a $\mathrm{PPE}^{8}$, a lesão da barreira hematoencefálica (BHE) não é tão pronunciada como em outros modelos ou como no próprio TCE em humanos ${ }^{9}$, o que é uma característica desvantajosa desse modelo.

O segundo modelo consiste no estabelecimento de lesão encefálica focal obtida por meio de resfriamento intenso (lesão criogênica). Nesse modelo observam-se alterações histopatológicas do encéfalo ${ }^{10}$ como ruptura da BHE, formação de edema, hemorragia intraparenquimatosa e importante prejuízo no mecanismo de $\mathrm{ARVE}^{5}$, promovendo incremento da volemia intravascular em decorrência da estase resultante ${ }^{8}$. Todavia, o efeito de massa, como observado no modelo de balão, é incipiente.

Nos modelos de lesão criogênica, a ruptura da BHE e o edema resultante parecem contribuir de forma mais importante para redução da CE do que nos modelos de balão, onde o efeito de massa parece ser o principal componente que reduz a ARVE e assim, altera a CE.

Ambos os modelos têm sido freqüentemente empregados no estudo do TCE sem levar tais considerações em conta e ainda não foram confrontados para comparação das alterações na ARVE e na CE, particularmente, na vigência de hipertensão arterial concomitante a HIC, e, portanto, carecem de meIhor esclarecimento se são de fato comparáveis.

Por essas razões, o objetivo do presente estudo é comparar o efeito da hipertensão arterial induzida sobre a complacência e a pressão de perfusão do encéfalo em dois modelos experimentais empregando cães, sejam submetidos à lesão encefálica causada por insuflação de balão subdural ou por lesão criogênica com nitrogênio líquido.

\section{MÉTODO}

Dezoito cães de ambos os sexos, sem raça definida, pesando de $10 \mathrm{~kg}$ a $20 \mathrm{~kg}$, em jejum de 10 horas, sem medicação pré-anestésica e após exame e liberação do veterinário responsável pelo biotério da instituição foram colocados em caixa acrílica transparente saturada e ventilada com halotano a $4 \%$ em fluxo de 10 L. $\min ^{-1}$ de $\mathrm{O}_{2} / \mathrm{N}_{2} \mathrm{O}$ (1:1).

Após inconsciência e redução da freqüência respiratória para 12 i.r.p.m. posicionamento supino em goteira de Claude-Bernard, intubação traqueal, redução da fração inspirada de halotano para $2 \%$, instalação de ventilação controlada mecânica com volume corrente de $8 \mathrm{~mL} \cdot \mathrm{kg}^{-1}$, relação I/E de 1:1 e freqüência ajustada para manter $\mathrm{CO}_{2}$ expirado em $33 \pm$ $2 \mathrm{mmHg}$. Colocação do sensor do oxímetro de pulso na língua, eletrocardioscopia na derivação $D_{\|}$nas patas, termômetro timpânico à direita, capnógrafo e analisador de gases. Infiltração subcutânea com $1 \mathrm{~mL}$ de lidocaína a $1 \%$ prévia a todas incisões. Dissecção e cateterização da artéria femoral para monitorização contínua da pressão arterial média (PAM) e da veia femoral para pressão venosa central (PVC)à direita. Dissecção e cateterização da artéria femoral esquerda para coleta de amostra sangüínea para dosagens bioquímicas e da veia femoral ipsilateral para infusão de anestésicos e solução fisiológica a 0,9\%. Redução da fração inspirada de halotano para $1 \%$ e administração de fentanil $2 \mu \mathrm{g} \cdot \mathrm{kg}^{-1}$ 
e pancurônio $0,08 \mathrm{mg} \cdot \mathrm{kg}^{-1}$. Mudança de decúbito para posição de "esfinge". Exposição cirúrgica da calota craniana. Trepanação circular de $1 \mathrm{~cm}$ de diâmetro, $1,5 \mathrm{~cm}$ lateralmente à linha média do crânio e introdução de sensor intracerebral para monitorização da PIC.

Distribuição aleatória, em dois grupos com o mesmo número de animais, segundo o esquema:

1 - Grupo balão subdural (BS) - Trepanação de $3 \mathrm{~mm} \times 5$ $\mathrm{mm}$ e abertura da dura-máter, no hemisfério contralateral ao cateter de PIC, introdução de $2 \mathrm{~cm}$ da extremidade distal da sonda de Foley no espaço subaracnóideo. Fechamento hermético com resina acrílica. Insuflação lenta e gradual do balonete com solução de $\mathrm{NaCl}$ a $0,9 \%$ para PIC em torno de $40 \mathrm{mmHg}$ por 20 minutos (Figuras 1 e 2).

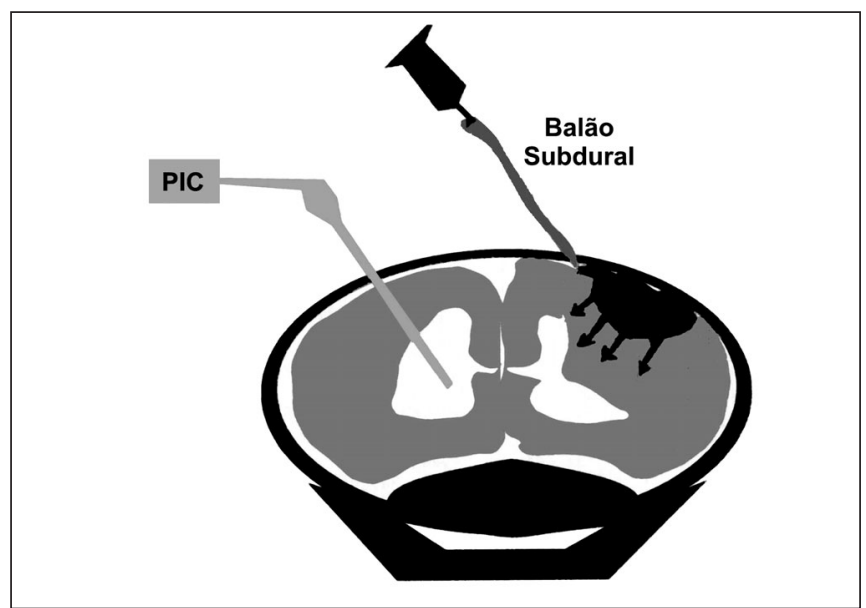

Figura 1 - Representação Esquemática do Grupo BS

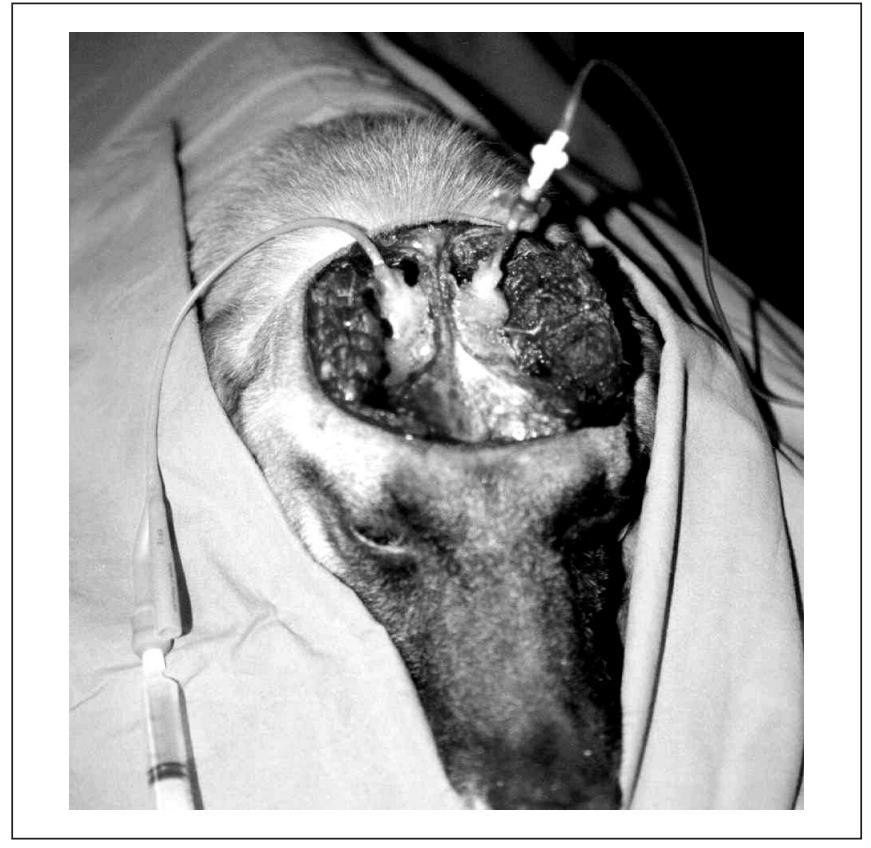

Figura 2 - Preparação Experimental do Grupo BS
2 - Grupo lesão criogênica (LC) - Fixação de funil plástico com resina acrílica à calota craniana contralateral à trepanação da PIC. A área delimitada pela extremidade do funil fixada na calota craniana foi uma circunferência de $25 \mathrm{~mm}$ de diâmetro. A seguir, nitrogênio líquido foi vertido dentro do funil, diretamente sobre a tábua óssea por 20 minutos (Figuras 3 e 4).

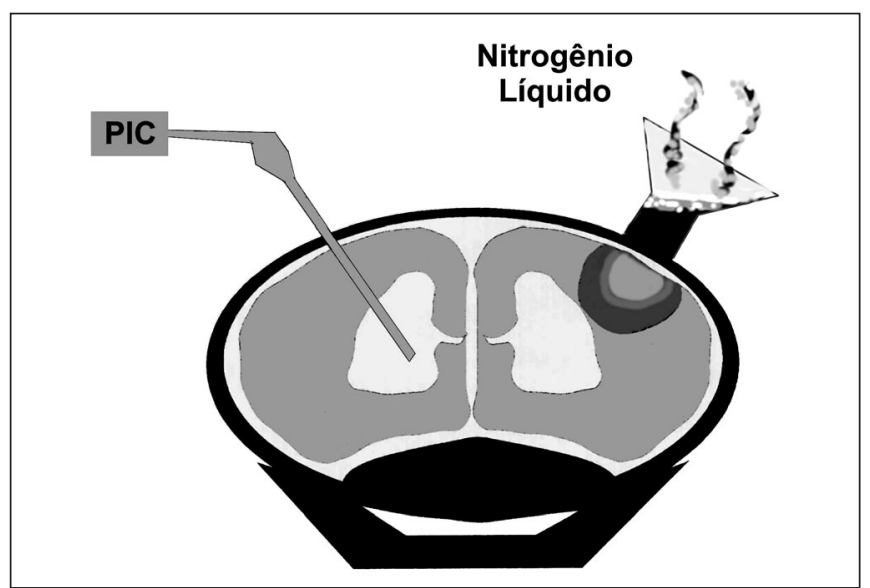

Figura 3 - Representação Esquemática do Grupo LC

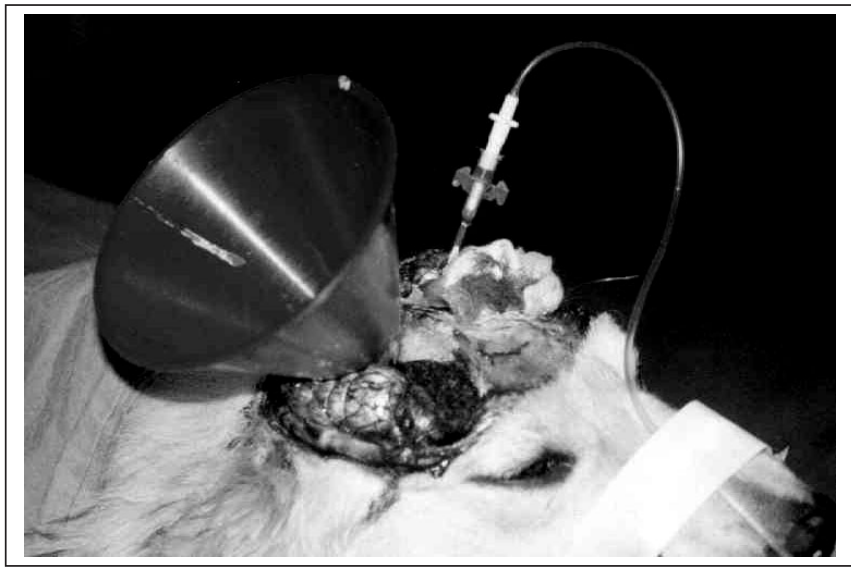

Figura 4 - Preparação Experimental do Grupo LC

Teste de complacência encefálica: o teste de complacência encefálica constitui o cerne do modelo experimental proposto neste estudo e depende do estado funcional do mecanismo autorregulatório vascular do encéfalo. Consiste na elevação controlada da PAM de um valor basal em torno de 90 $\mathrm{mmHg}$ até o limite de $140 \mathrm{mmHg}$ e na mensuração da PIC e PPE resultantes.

O volume sangüíneo encefálico (VSE) é função do FSE. Assim, a elevação da pressão arterial dentro desses limites de pressão pouco modifica o volume intracraniano se o mecanismo de ARVE está íntegro, e se o faz, é para um valor discretamente menor; portanto, essa hipertensão induzida não eleva a PIC ${ }^{5}$. De modo oposto, se o encéfalo sofre agressão 
e o mecanismo de autorregulação torna-se funcionalmente comprometido $^{5}$, o aumento da pressão arterial, mesmo dentro do intervalo fisiológico de pressão arterial, dilata passivamente as arteríolas de resistência e aumenta o FSE e, por consecutivo, o VSE ${ }^{8}$. Este incremento no volume intracraniano termina por elevar a PIC e reduzir a complacência do encéfalo ${ }^{2}$.

A partir dessa conclusão pode-se inferir qualitativamente o estado funcional da autorregulação vascular ${ }^{16}$ e quantificar a CE por meio da variação da PAM dentro do intervalo fisiológico desse fenômeno.

Quando o teste é negativo, ou seja, quando não há alteração significativa da PIC, infere-se que o mecanismo de ARVE esteja íntegro (curva "a" da figura 5) e que a elasticidade do parênquima esteja na região linear da curva de complacência encefálica (segmento "a" da figura 6).

Quando o teste é positivo, isto é, quando ocorre elevação significativa da PIC com aumento da PAM, infere-se que exista prejuízo funcional na ARVE (curva "b" da figura 5) e que a complacência encefálica encontre-se parcialmente diminuí-

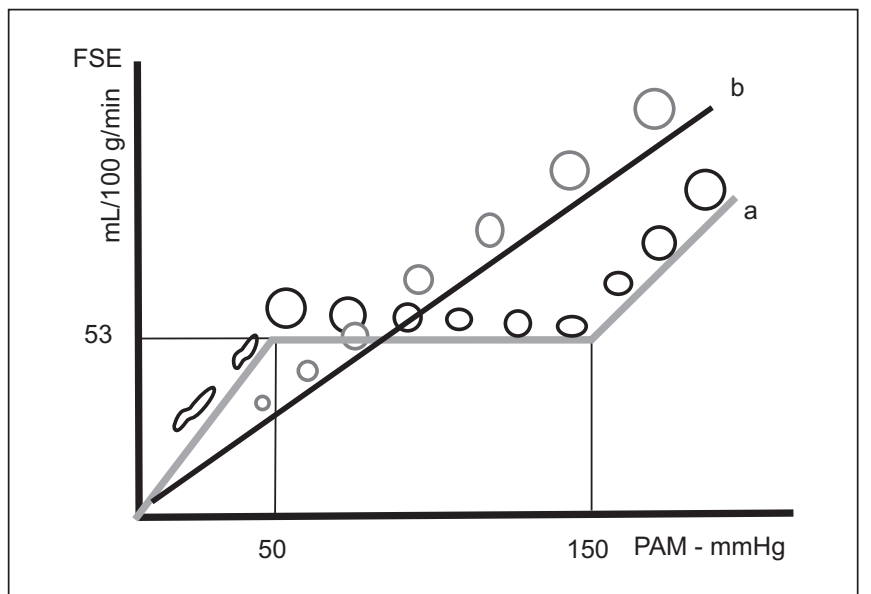

Figura 5 - Fluxo Sangüíneo Encefálico em Função da Pressão Arterial Média. As circunferências representam o calibre da vasculatura de resistência

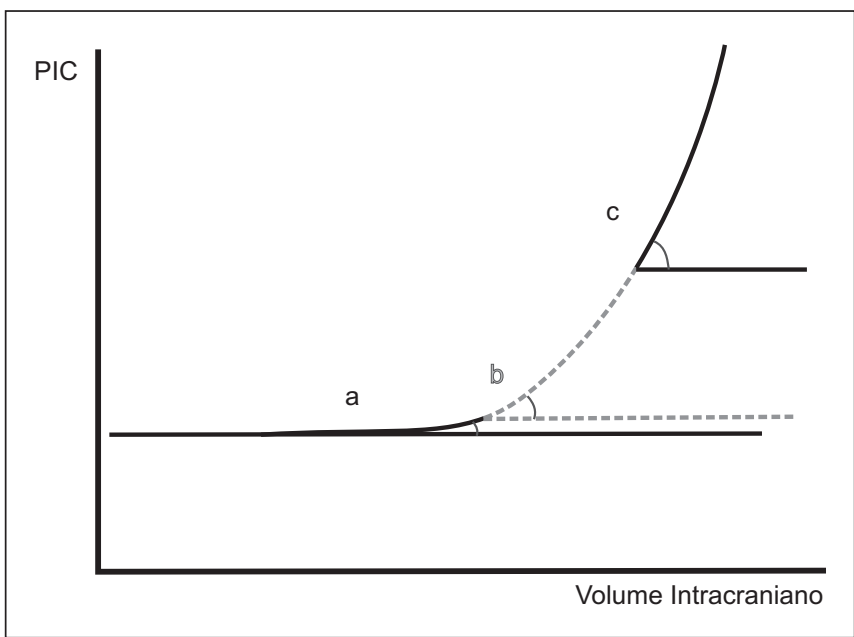

Figura 6 - Pressão Intracraniana em Função do Volume Intracraniano da na HIC moderada (segmento "b") ou muito reduzida na HIC grave (segmento "c") da curva de Langfitt $^{8}$ (Figura 6).

A eventual variação do VSE é função da atividade da ARVE, mas quando ocorre, também é dependente da magnitude dessa variação pressórica. Se a variação da PAM for sempre de mesma magnitude e se todas as outras condições que interfiram com o fluxo sangüíneo encefálico forem mantidas constantes, pode-se considerar o incremento observado na PIC como dependente apenas do estado funcional da ARVE e do grau de complacência do encéfalo.

Assim, considerando que: 1) as elevações de PAM sejam arbitrárias e definidas pelos pesquisadores, e, portanto, semeIhantes nos diversos momentos do estudo; 2) que as hipertensões induzidas (PAM final menos inicial) terminem por gerar incrementos do volume sangüíneo encefálico também semelhantes, proporcionais à intensidade dessa variação de PAM; e 3) que o aumento da PIC seja decorrente apenas desses incrementos volumétricos de sangue dentro do crânio, então, pode-se estabelecer uma relação entre a variação da PIC em função da variação da PAM, ou seja, uma relação absoluta de pressão em termos de pressão.

A variação da PAM foi arbitrariamente determinada em 50 $\mathrm{mmHg}$ por ser valor intenso o suficiente para poder elevar a PIC em caso de incompetência da ARVE e ser obtida por meio da infusão titulada e controlada de solução diluída de norepinefrina. Partiu-se de um valor basal em torno de 90 $\mathrm{mmHg}$ para um teto em torno de $140 \mathrm{mmHg}$, com o cuidado de não ultrapassar o limite superior do fenômeno autorregulatório. Também procurou-se partir rigorosamente do mesmo valor basal de PAM e repetir cada uma das elevações da PAM sempre no mesmo intervalo de tempo.

As variáveis analisadas - PAM, PIC e PPE - foram coletadas nos seguintes momentos:

(M0) - Momento zero - após período de estabilização, correspondente a 20 minutos após inserção dos sensores para as variáveis estudadas. Neste tempo também foram analisadas as dosagens de sódio (osmolaridade), $\mathrm{pH}, \mathrm{PaCO}_{2}, \mathrm{PaO}_{2}$, saturação de $\mathrm{O}_{2}$ (ARVE) e hematócrito (viscosidade) do sangue arterial, para verificar se os grupos eram semelhantes nessas variáveis.

(M1) - Momento 1 - Primeiro teste de complacência encefálica: infusão titulada de norepinefrina até PAM alvo e registro da PIC e PPE resultantes - correspondente à resposta do encéfalo (ARVE e CE) normal frente à hipertensão arterial induzida.

(M2) - Momento 2 - Quinze minutos após o final da infusão de norepinefrina em M1, quando as variáveis hemodinâmicas já haviam retornado aos valores basais, iniciou-se a lesão encefálica: insuflação do balão subdural até atingir PIC acima de $30 \mathrm{mmHg}$ no grupo BS ou início da lesão criogênica, vertendo o nitrogênio líquido no funil por um período de 20 minutos no grupo LC.

(M3) - Momento 3 - Após 20 minutos de balão subdural insuflado no grupo BS com PIC mínima de $30 \mathrm{mmHg}$ e 20 minutos após o término da lesão criogênica, ou seja, 40 
minutos após o início da lesão criogênica no grupo LC, correspondente ao final do estabelecimento das lesões nos grupos.

(M4) - Momento 4 - Segundo teste de complacência: infusão titulada de norepinefrina até PAM alvo e registro da PIC e PPE resultantes - correspondente à resposta do encéfalo (ARVE e CE) lesado frente à hipertensão arterial induzida. A figura 7 mostra a cronologia da experimentação.

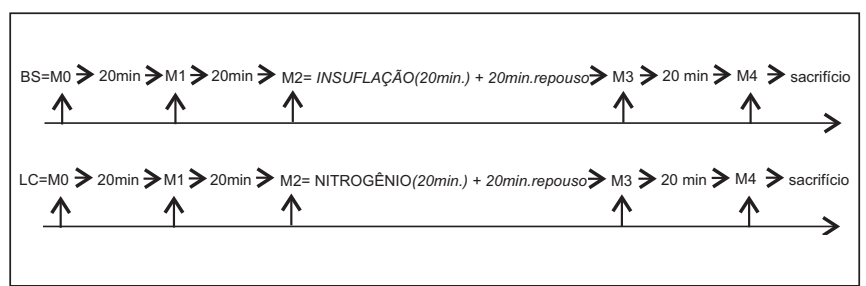

Figura 7 - Representação Cronológica da Preparação Experimental

Ao término do experimento, houve aprofundamento do plano anestésico seguido do sacrifício com injeção em bolus de 10 $\mathrm{mL}$ de cloreto de potássio a 19,1\%. A análise estatística empregou: o teste $t$ de Student pareado para comparar as variações observadas dentro do mesmo grupo entre os momentos $M 0$ e $M 1$, sem que o encéfalo sofresse agressão; o teste $t$ de Student para duas amostras distintas para comparar os dois grupos no momento M0 (pressão normal) e em M1 (hipertensão arterial) para verificar se os animais pertenciam à mesma amostra populacional; o teste $t$ de Student para duas amostras distintas para comparar as variáveis entre os grupos nos seguintes momentos:

1. M2 quando se iniciou a insuflação do balão para atingir o valor de PIC entre 30 e 50 mmHg no grupo balão subdural e início do resfriamento no grupo lesão criogênica.

2. M3 quando completados 20 minutos de balão insuflado no grupo balão subdural e depois de 40 minutos após iniciar o resfriamento no grupo lesão criogênica.

3. M4 após a infusão da segunda dose de norepinefrina para causar hipertensão arterial em ambos os grupos.

Acomparação dentro do grupo usou o teste $t$ de Student pareado nos seguintes tempos:

1. Entre M2 e M3 para averiguar se houve alteração entre estes tempos, pois os mecanismos de lesão foram distintos nos dois grupos.

2. Entre M3 e M4 para averiguar se a infusão de norepinefrina causou alteração em cada tipo específico de lesão.

\section{RESULTADOS}

Os animais dos dois grupos pertenceram à mesma população quando avaliados pelas variáveis hemodinâmicas (pressão arterial média e freqüência cardíaca), encefálicas (PIC e PPE), metabólicas (sódio, potássio, pressão parcial arterial de gás carbônico, hematócrito e temperatura) e morfométrica (peso) no momento M0 (Tabelas I, II e III).

Tabela I - Variáveis Bioquímicas $\left(\mathrm{Na}^{+}, \mathrm{K}^{+}\right.$, Hematócrito e Pressão Parcial Arterial de $\mathrm{CO}_{2}$ ) e Morfométrica (peso) dos Grupos BS e LC no Momento M0

\begin{tabular}{lccc}
\hline Variáveis & $\mathrm{BS}{ }^{*}$ & $\mathrm{LC}{ }^{*}$ & $\mathrm{p}$ \\
\hline Peso $(\mathrm{kg})$ & $14,2 \pm 2,8$ & $12,5 \pm 1,7$ & 0,67 \\
$\mathrm{Na}+\left(\mathrm{mEq} \cdot \mathrm{L}^{-1}\right)$ & $145,2 \pm 1,5$ & $144,6 \pm 2,1$ & 0,81 \\
$\mathrm{~K}+\left(\mathrm{mEq} \cdot \mathrm{L}^{-1}\right)$ & $3,8 \pm 0,3$ & $3,6 \pm 0,3$ & 0,04 \\
Hematócrito $(\%)$ & $37,8 \pm 3,9$ & $39,7 \pm 6,8$ & 0,54 \\
PaCO $_{2}(\mathrm{mmHg})$ & $34,2 \pm 3,8$ & $34,3 \pm 3,5$ & 0,65 \\
\hline
\end{tabular}

*Valores expressos em Média \pm DP

Pressão arterial média (PAM): Em M1, houve aumento da PAM nos grupos BS $(145,7 \pm 6,2 ; p<0,05)$ e em LC $(140,7 \pm$ $11,5 ; p<0,05)$. Além disso, a intensidade da hipertensão arterial induzida $(\mathrm{HAl})$ foi semelhante nos dois grupos nesse tempo do estudo $(p=0,25)$, como era desejado. Após o período de repouso e estabilização, o que corresponde ao início da lesão nos dois grupos, em M2, a PAM retornou aos valores basais nos dois grupos. Do mesmo modo, a PAM de repouso até o início do segundo teste de complacência em M3 também foi semelhante nos dois grupos ( $B S=97 \pm 7,1$ e $L C=91,8$ $\pm 10,3 ; p=0,31)$. Após estabelecimento da lesão em $M 3$, a PAM permaneceu semelhante a M2 em BS $(101,3 \pm 8,1 ; p=$ $0,65)$ e em LC $(95,8 \pm 9,4 ; p=0,41)$. Como esperado, durante HAl houve aumento significativo da PAM, tanto em BS (148,1 $\pm 10 ; p<0,001)$ como em LC $(138,4 \pm 8 ; p<0,001)$. Neste último teste a PAM de BS foi maior, ainda que discretamente, que em LC $(p=0,04)$. (Tabela II e Figura 8).

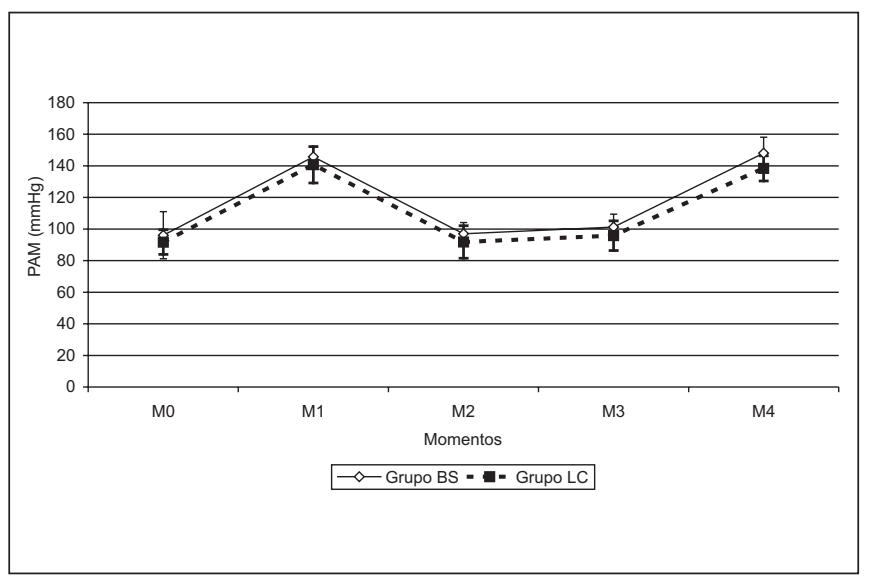

Figura 8 - Pressão Arterial Média dos Grupos BS e LC nos Momentos M0, M1, M2, M3 e M4 
Tabela II - Pressão Arterial Média (PAM) dos Grupos BS e LC nos Momentos M0, M1, M2, M3 e M4

\begin{tabular}{lcccccc}
\hline Momentos & $\begin{array}{c}\text { BS } \\
\text { Média } \pm \text { DP }\end{array}$ & $\begin{array}{c}\text { Média } \pm \text { DP } \\
\text { M0 }\end{array}$ & $\begin{array}{c}\text { BS X LC } \\
\text { Significância }\end{array}$ & Intervalo & $\begin{array}{c}\text { BS X BS } \\
\text { Significância }\end{array}$ & $\begin{array}{c}\text { LC X LC } \\
\text { Significância }\end{array}$ \\
\hline M1 & $96,1 \pm 14,9$ & $91,7 \pm 7,8$ & 0,32 & & & \\
M2 & $145,7 \pm 6,2$ & $140,7 \pm 11,5$ & 0,25 & M0 - M1 & $<0,001$ & $<0,001$ \\
M3 & $97 \pm 7,1$ & $91,8 \pm 10,3$ & 0,31 & M1 - M2 & $<0,001$ & $<0,001$ \\
M4 & $101,3 \pm 8,1$ & $95,8 \pm 9,4$ & 0,19 & M2 - M3 & 0,06 & 0,41 \\
\hline
\end{tabular}

Valores em mmHg expressos em Média \pm DP; BS = Balão subdural; LC = Lesão criogênica

Tabela III - Pressão Intracraniana (PIC) dos Grupos BS e LC nos Momentos M0, M1, M2, M3 e M4

\begin{tabular}{|c|c|c|c|c|c|c|}
\hline Momentos & $\begin{array}{c}\text { BS } \\
\text { Média } \pm \text { DP }\end{array}$ & $\begin{array}{l}\text { LC } \\
\text { Média } \pm \text { DP }\end{array}$ & $\begin{array}{c}\text { BS X LC } \\
\text { Significância }\end{array}$ & Intervalo & $\begin{array}{c}\text { BS X BS } \\
\text { Significância }\end{array}$ & $\begin{array}{c}\text { LC X LC } \\
\text { Significância }\end{array}$ \\
\hline M0 & $8,2 \pm 4$ & $9,4 \pm 3,6$ & 0,27 & & & \\
\hline M1 & $9,5 \pm 4,7$ & $11,3 \pm 3,7$ & 0,21 & M0 - M1 & 0,05 & 0,01 \\
\hline M2 & $37 \pm 7,7$ & $12 \pm 3,7$ & $<0,001$ & M1 - M2 & $<0,001$ & 0,18 \\
\hline M3 & $32 \pm 12,8$ & $26 \pm 6,6$ & 0,39 & M2 - M3 & 0,36 & $<0,001$ \\
\hline M4 & $38 \pm 6,7$ & $35,7 \pm 6,7$ & 0,98 & M3 - M4 & 0,12 & $<0,001$ \\
\hline
\end{tabular}

Valores em mmHg expressos em Média \pm DP; BS = Balão subdural; LC = Lesão criogênica

Pressão intracraniana (PIC): Em M1, houve aumento significativo da PIC tanto em BS $(9,5 \pm 4,7 ; p=0,04)$ como em LC $(11,3 \pm 3,7 ; p=0,01)$, de magnitude semelhante nos dois grupos $(p=0,21)$. Em M2 houve aumento significativo da PIC em BS $(37 \pm 7,4 ; p=0,01)$ com a insuflação do balão, enquanto em LC a PIC manteve-se inalterada $(12 \pm 3,7 ; p=0,18)$. Neste instante os valores da PIC tornaram-se distintos $(p<0,001)$. Em M3, a PIC manteve-se elevada no grupo BS, sem diferença em relação a M2 (32 $\pm 12,9$ e $p=0,39)$. Já no grupo LC, vinte minutos após a lesão criogênica, o que marca o tempo do estudo M3, a PIC aumentou significativamente $(26 \pm 6,6 ; p<$ $0,001)$, tornando as PIC novamente semelhantes $(p=0,39)$. Em M4, no segundo teste de complacência, o aumento observado no grupo BS não foi significativo $(38 \pm 17,1 ; p=0,12)$, por sua vez, no grupo LC o aumento foi significativo $(35,7 \pm$

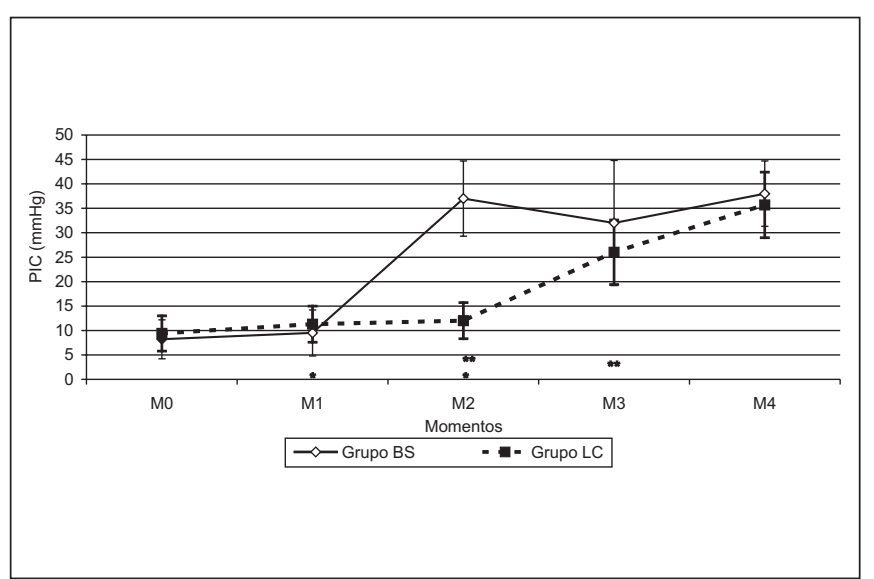

Figura 9 - Pressão Intracraniana (PIC) nos Grupos BS e LC nos Momentos M0, M1, M2, M3 e M4
$6,7 ; p<0,001)$, embora os valores da PIC fossem semelhantes nos grupos ( $p>0,98)$ (Tabela III e Figura 9). Pressão de perfusão encefálica (PPE): Houve aumento significativo da PPE entre M0 e M1, tanto em BS (136 $\pm 6,9 ; p<$ $0,001)$ como em LC $(126 \pm 5 ; p<0,001)$, maior no grupo BS $(p$ $=0,002)$. Já em M2, houve redução da PPE em BS $(60 \pm 6,9 ; p$ $<0,001)$ e em LC $(79,8 \pm 10,2 ; p<0,001)$. A redução da PPE em BS foi maior que em LC $(p<0,001)$. Em M3, como houve aumento da PPE em BS $(72,6 \pm 13 ; p=0,02)$ e redução em $L C$ $(69,8 \pm 9,9 ; p=0,01)$, a PPE tornou-se semelhante nos dois grupos $(p=0,43)$. Com a HAl em M4, a PPE aumentou em BS $(110,1 \pm 17,1 ; p<0,001)$ e em LC $(102,6 \pm 8,3 ; p<0,001)$. Esse incremento foi semelhante nos grupos BS e LC ( $p=$ 0,16), (Tabela IV e Figura 10).

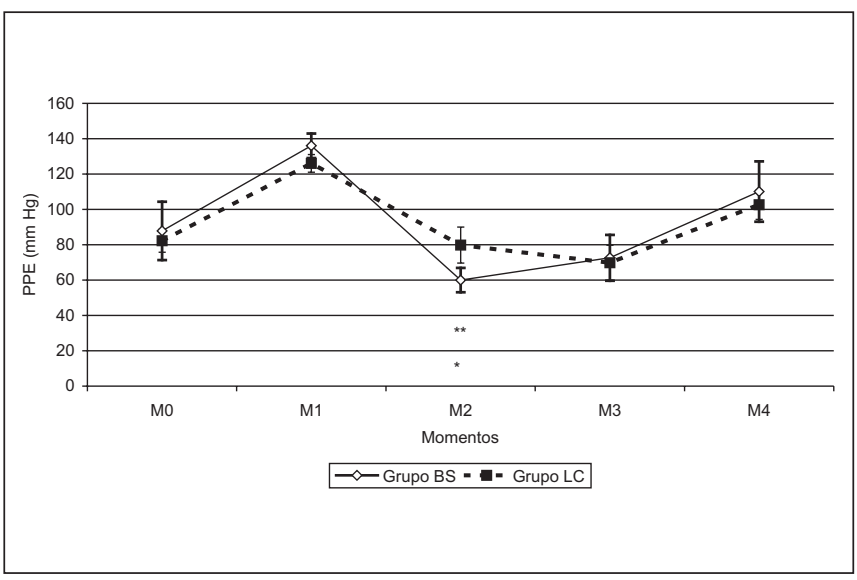

Figura 10 - Pressão de Perfusão Encefálica (PPE) dos Grupos BS e LC nos Momentos M0, M1, M2, M3 e M4

Revista Brasileira de Anestesiologia Vol. 55, № 3, Maio - Junho, 2005 
Tabela IV - Pressão de Perfusão Encefálica (PPE) dos Grupos BS e LC nos Momentos M0, M1, M2, M3 e M4

\begin{tabular}{|c|c|c|c|c|c|c|}
\hline Momentos & $\begin{array}{c}\text { BS } \\
\text { Média } \pm \text { DP }\end{array}$ & $\begin{array}{c}\text { LC } \\
\text { Média } \pm \text { DP }\end{array}$ & $\begin{array}{c}\text { BS X LC } \\
\text { Significância }\end{array}$ & Intervalo & $\begin{array}{c}\text { BS X BS } \\
\text { Significância }\end{array}$ & $\begin{array}{l}\text { LC X LC } \\
\text { Significância }\end{array}$ \\
\hline MO & $87,8 \pm 16,5$ & $82,3 \pm 6,5$ & 0,19 & & & \\
\hline M1 & $136 \pm 6,9$ & $126 \pm 5$ & 0,002 & M0 - M1 & $<0,001$ & 0,02 \\
\hline M2 & $60 \pm 6,9$ & $79,8 \pm 10,2$ & $<0,001$ & M1 - M2 & $<0,001$ & $<0,001$ \\
\hline M3 & $72,6 \pm 13$ & $69,8 \pm 9,9$ & 0,43 & M2 - M3 & 0,03 & 0,01 \\
\hline M4 & $110,1 \pm 17,1$ & $102,6 \pm 8,3$ & 0,16 & M3 - M4 & $<0,001$ & $<0,001$ \\
\hline
\end{tabular}

Valores em mmHg expressos em Média \pm DP; BS = Balão subdural; LC = Lesão criogênica

Tabela V - Temperatura Timpânica dos Grupos BS e LC nos Momentos M0, M1, M2, M3 e M4

\begin{tabular}{lcccccc}
\hline Momentos & $\begin{array}{c}\text { BS } \\
\text { Média } \pm \text { DP }\end{array}$ & $\begin{array}{c}\text { LC } \\
\text { Média } \pm \text { DP }\end{array}$ & $\begin{array}{c}\text { BS } \times \text { LC } \\
\text { Significância }\end{array}$ & Intervalo & $\begin{array}{c}\text { BS X BS } \\
\text { Significância }\end{array}$ & $\begin{array}{c}\text { LC X LC } \\
\text { Significância }\end{array}$ \\
\hline M0 & $35,2 \pm 1,7$ & $34,5 \pm 1,2$ & 0,17 & & & 0,12 \\
M1 & $35,3 \pm 1,7$ & $34,5 \pm 1,3$ & 0,16 & M0 - M1 & 0,86 \\
M2 & $34,5 \pm 1,8$ & $33,5 \pm 1,4$ & 0,02 & M1 - M2 & 0,05 & 0,006 \\
M3 & $35,2 \pm 1,9$ & $33,4 \pm 1,7$ & 0,04 & M2 - M3 & 0,38 & 0,81 \\
M4 & $35,3 \pm 1,7$ & $33,5 \pm 1,6$ & 0,03 & M3 - M4 & 0,66 & 0,02 \\
\hline
\end{tabular}

BS = Balão subdural; LC = Lesão criogênica

Temperatura: A temperatura timpânica sofreu redução significativa em M2 nos dois grupos, BS $(35,4 \pm 1,8 ; p=0,05)$ e LC $(33,5 \pm 1,4 ; p=0,006)$, sendo menor em LC $(p=0,02)$, e assim se mantendo até M4 (Tabela V).

\section{DISCUSSÃO}

Vários modelos de agressão encefálica controlada têm sido utilizados para estudar as modificações que ocorrem durante e após o trauma cranioencefálico ${ }^{9,20,29,31-35}$. Intuitivamente, o modelo que mais se aproxima desta forma de agressãoé a contusão do crânio com objetos sólidos.

Entretanto, este tipo de agressão freqüentemente gera hematomas intracranianos e fraturas do estojo ósseo, fatores que exercem efeitos imprevisíveis sobre a complacência cranioencefálica ${ }^{17}$. Por isso, modelos experimentais que possibilitam um aumento gradual e controlado da PIC, mais previsíveis, portanto, são os mais empregados.

A insuflação com balão subdural ou peridural, infusão de líquido intraventricular ou outras formas de expansão volumétrica intracraniana podem ser mais previsíveis quanto ao valor e tempo que se deseje manter a hipertensão intracraniana. Porém, não comprometem muito a permeabilidade da barreira hematoencefálica e, por conseqüência, o edema do tecido nervoso parece não ser um fator importante nesses modelos. Alguns autores ${ }^{18}$ sugerem que, após o TCE, o edema do encéfalo contribua para aumentar o volume dentro do crânio mais do que a estase sangüínea decorrente da vasoparesia, ou seja, que o aumento da PIC possa ser decorrente muito mais do aumento de água, seja no extravascular, seja no intracelular, do que de sangue intravascular.

Além disso, no trauma cranioencefálico grave, freqüentemente a PIC está aumentada num valor crítico, a ponto de comprometer a perfusão encefálica ${ }^{6}$. Esta hipertensão intracraniana extremamente elevada nem sempre foi encontrada nos estudos que avaliam a PIC ${ }^{17}$, e que portanto, nem sempre tiveram a pressão de perfusão encefálica reduzida a esses níveis críticos que comprometessem a reatividade vascular e o fluxo sangüíneo encefálico.

Neste modelo, no grupo BS procurou-se manter a PIC acima de $30 \mathrm{mmHg}$, como encontrado em traumas cranioencefálicos graves, valor que reduziu significativamente a pressão de perfusão encefálica, de um valor basal médio de $87 \mathrm{mmHg}$ em M0 para 60 mmHg em M2 ( $p<0,001)$. Com hipertensão intracraniana intensa o suficiente, além de reduzir a perfusão, ocorre também redução do tônus muscular das arteríolas encefálicas e importante prejuízo funcional no mecanismo autorregulatório, conduzindo à dilatação passiva do leito vascular de resistência e aumento do volume sangüíneo encefálico na vigência de hipertensão arterial sistêmica ${ }^{8}$, mesmo que em valores diminutos, agravando ainda mais a hipertensão intracraniana já existente.

Um grupo ${ }^{6}$ mostrou que quando a pressão de perfusão encefálica é menor que $60 \mathrm{mmHg}$, o fluxo sangüíneo encefálico começa a reduzir-se. Por sua vez, outros autores ${ }^{16}$ demonstraram em cães anestesiados, se respeitados os limites de pressão arterial entre 50 e $150 \mathrm{mmHg}$, que a complacência do encéfalo relaciona-se à pressão de perfusão de modo diretamente proporcional em condições normais, isto é, com o encéfalo íntegro e autorregulação preservada; e de modo indiretamente proporcional quando o encéfalo sofre agressão que prejudica funcionalmente o seu mecanismo autorregulatório. Quando a autorregulação é normal, a variação da pressão arterial média dentro do intervalo fisiológico é incapaz de alterar significativamente o FSE, e, por conseguinte, de alterar também de forma significativa o volume sangüíneo encefálico (VSE) e modificar a complacência. Ao contrário, como mecanismo autorregulatório abolido, o VSE sofre incremento em razão do incremento do FSE quando a PAM se eleva, reduzindo a complacência. 
A avaliação da complacência encefálica por meios não volumétricos, isto é, sem a modificação do volume do compartimento intracraniano, foi realizada em estudos diversos ${ }^{19,29,30}$. Esses autores estudaram a morfologia da onda B da curva de PIC na presença de HIC, mas não encontraram correlação significativa da alteração morfológica dessa onda com a complacência encefálica avaliada pelos métodos volumétricos. Também sugeriram que o melhor método de avaliação seja o índice volume/pressão utilizado por outros autores ${ }^{2,20}$.

Durante o transporte, cirurgia ou permanência do paciente na unidade de terapia intensiva, com certa freqüência ele é submetido a estímulos nociceptivos que elevam a pressão arterial a valores indesejáveis, o que termina por reduzir a perfusão do neurônio, justamente quando o mecanismo de reatividade vascular do encéfalo está funcionalmente prejudicado.

Para tentar reproduzir essa situação a proposta deste estudo avaliou a complacência encefálica exatamente por meio da hipertensão arterial induzida, o que também aumenta a volemia intracraniana quando o encéfalo sofre agressão ${ }^{6,8}$.

A hipertensão intracraniana foi limitada no grupo BS em valores abaixo de $50 \mathrm{mmHg}$ para que se pudesse avaliar a complacência nos momentos subseqüentes ao estabelecimento da lesão.

A comparação da complacência dos modelos de balão com o de lesão criogênica foi importante, uma vez que não se sabia como este modelo iria se comportar com uma extensão de lesão antes não descrita.

Além disso, insuflação demasiada do balão subdural eventualmente deslocaria as estruturas adjacentes, causando hemorragias meníngeas, herniações, ingurgitamento do sistema venoso e hidrocefalia por obstrução da drenagem de líquor ${ }^{1}$.

O modelo de lesão criogênica tem sido utilizado para avaliar a permeabilidade da barreira hematoencefálica tanto pela análise de densidade gravimétrica como pela quantidade de água em exame anatomopatológicos da região agredida do encéfalo ${ }^{10,14}$. A lesão criogênica também causa alterações na reatividade vascular encefálica na área comprometida ${ }^{5}$. Um grupo demonstrou que o edema e inchaço encefálico surgem de modo significativo já nos primeiros 15 a 30 minutos após lesão criogênica ${ }^{10}$. Entretanto, a maioria dos experimentos com lesão criogênica não produziu lesão tecidual de extensão suficiente para elevar a HIC em valores que comprometessem a pressão de perfusão encefálica, nem prejudicar suficientemente o mecanismo de ARVE, pois, ou a superfície resfriada tinha pequena extensão, ou pouco tempo de contato do agente criogênico com o cérebro ${ }^{11-15}$

Historicamente a lesão criogênica não tem sido utilizada para o estudo da PIC ${ }^{11,12,14}$, pois parece que a extensão da lesão descrita por esses autores não teve volume suficiente para comprometer a PIC e o mecanismo de ARVE de modo significativo. Como o volume comprometido que descrevem não é grande, parece que os mecanismos de compensação da complacência do encéfalo, deslocamento de líquor e sangue, são eficientes para manter a PIC em valores normais ou próximos deles $^{8,12}$.
No presente estudo a proposta foi avaliar a complacência encefálica empregando dois modelos distintos, porém, em ambos, o grau de agressão proposto foi maior do que o normalmente estudado, mais próximo aquele observado nos pacientes vítimas de TCE grave.

$\operatorname{Em~M0}$, as variáveis PAM $(p=0,31), \operatorname{PIC}(p=0,27)$ e PPE $(p=$ 0,19 ) foram semelhantes nos dois grupos, confirmando pertencerem à mesma população. Outras variáveis que também poderiam influenciar fluxo sangüíneo encefálico ${ }^{36}$, e, por conseguinte, nos resultados - temperatura central (metabolismo neuronal, $p=0,17$ ), sódio plasmático (edemas de origem osmótica, $p=0,81$ ), hematócrito (viscosidade sangüínea, $p=0,65$ ) e pressão arterial parcial de $\mathrm{CO}_{2}$ (alteração do mecanismo autorregulatório, $p=0,37$ ) - também foram analisadas. De modo análogo, não foram observadas diferenças significativas entre os dois grupos.

Tomou-se especial cuidado em não elevar a pressão arterial acima do limite superior da ARVE, (> $150 \mathrm{mmHg}$ ), para evitar que a hipertensão arterial atuasse sobre a ARVE per se ${ }^{21}$, ou aumentasse a permeabilidade capilar do encéfalo normal ${ }^{21-23}$.

O plano anestésico foi mantido em profundidade adequada, mas não demasiada, com a fração expirada de agente halogenado abaixo de $1 \%$ (menor que a DE95) devido à associação de opióide venoso em doses iguais nos dois grupos. Evitou-se ainda a hipercarbia para que disritmias cardíacas não ocorressem com a infusão de norepinefrina associada ao halotano ${ }^{24}$ nem causasse vasodilatação da vasculatura encefálica com conseqüente incremento do volume de sangue dentro do crânio ${ }^{25}$.

Neste trabalho houve um aumento limítrofe de significância da PIC em M1 nos dois grupos ( $p=0,05$ em BS e $p=0,01$ em LC), Entretanto, esse aumento foi discreto e bastante fugaz, sem significado biológico correspondente. Igualmente importante, não se observou diferença significativa entre os grupos na intensidade dessa variação $(p=0,21)$. Considerou-se normal esse breve incremento da PIC durante hipertensão arterial induzida. O aumento da $\mathrm{PIC}$ em resposta à hipertensão arterial em encéfalo normal, discreto e temporário, também foi observado por outros pesquisadores ${ }^{26,31}$. Em M1 a pressão de perfusão aumentou mais no grupo BS do que no grupo LC ( $p=0,002)$, provavelmente, porque o aumento da pressão arterial foi maior e ocorreu, concomitantemente, aumento de menor intensidade da PIC no grupo BS do que no grupo LC.

No grupo BS em M2, houve aumento repentino da PIC $(p<$ 0,001 ) e redução da pressão de perfusão encefálica ( $p<$ 0,001 ) como foi proposto, sendo mantido por 20 minutos nesses valores com insuflação complementar do balão subdural cada vez que a PIC tendesse a diminuir, para que pudesse ocorrer certo grau de comprometimento da reatividade vascular encefálica. Após esse período de agressão e redução da pressão de perfusão, observou-se em M3 um aumento discreto da pressão arterial nos dois grupos, embora esse incremento não tenha sido significativo $(p=0,19)$.

A elevação da pressão arterial observada no repouso dos dois grupos pode ser explicada como sendo resposta do sis- 
tema nervoso central à isquemia gerada pela hipertensão intracraniana $^{27,28}$.

Não houve redução da PIC no grupo BS entre M2 e M3 ( $p=$ $0,36)$. Embora não significativa, a discreta redução da PIC provavelmente deveu-se aos mecanismos de compensação da complacência, deslocamento de líquor e sangue para fora do crânio enquanto o balão subdural era desinsuflado.

Ainda que não representem diferenças significativas quando considerados isoladamente, o aumento da pressão arterial associado à redução da PIC resultou em aumento importante da pressão de perfusão encefálica em M3 no grupo BS ( $p=$ $0,03)$.

Em M4 o significativo aumento da pressão arterial média produzido ( $p<0,001$ em BS e em LC) correspondeu ao esperado, pois o efeito dessa hipertensão arterial induzida sobre a PIC do encéfalo agredido foi justamente o objetivo deste estudo.

Nesse momento no grupo BS, embora tenha sido observado aumento da PIC, esse aumento não foi significativo $(p=0,12)$ quando comparada ao momento anterior M3, quando a pressão arterial era normal, nesse mesmo grupo. A resultante foi um aumento da pressão de perfusão encefálica $(p<0,001)$ com a infusão da norepinefrina.

Talvez uma agressão mais intensa do encéfalo fosse necessária no grupo BS para que houvesse maior comprometimento da ARVE. O tempo no qual o balão esteve insuflado poderia ser superior a 20 minutos ou o nível da hipertensão intracraniana poderia ser maior que $50 \mathrm{mmHg}$.

Por outro lado, a isquemia de 20 minutos parece ter sido razoável, e a insuflação do balão mantendo a PIC acima de 50 $\mathrm{mmHg}$ poderia deslocar as estruturas encefálicas adjacentes e modificar a dinâmica do sangue e líquor.

Por sua vez, no grupo LC em M2, mesmo já iniciada a lesão com nitrogênio líquido, a PIC ainda não mostrava nenhuma alteração $(p=0,18)$, provavelmente porque as alterações sobre a barreira hematoencefálica e na reatividade vascular encefálica ainda não tivessem tido tempo de se instalar. Dois autores demonstraram que, tanto o edema como o inchaço encefálico manifestam-se na lesão criogênica do encéfalo após período tão curto como 15 a 20 minutos após estabelecimento da lesão, continuando a evoluir por até 12 horas após o início da reperfusão do tecido lesado ${ }^{10}$.

Como neste momento a PAM foi semelhante ao valor basal inicial $(p=0,93)$ e também não foi diferente da PAM do grupo BS $(p=0,31)$, a PPE do grupo LC foi maior que o do grupo BS $(p<0,001)$.

No momento M3, quarenta minutos após o início da lesão criogênica, correspondentes a 20 minutos de lesão acrescidos de 20 minutos de repouso para o degelo e reperfusão do encéfalo super-resfriado, a PIC começou a aumentar $(p<$ $0,001)$. Nesse momento as repercussões na PIC e no mecanismo reativo da vasculatura encefálica causados pela lesão criogênica já se manifestavam, em concordância com os achados desses autores ${ }^{10}$.

Com esse aumento da PIC, a PPE sofreu redução $(p=0,01)$ nesse grupo (LC). No mesmo momento M3, a PPE dos gru-

Revista Brasileira de Anestesiologia

Vol. 55, № 3, Maio - Junho, 2005 pos BS e LC foi semelhante ( $p=0,43)$. Em M4 a infusão de norepinefrina elevou a PAM ( $p<0,001)$ que causou aumento da PPE $(p<0,001)$, mas também aumentou a PIC $(p<0,001)$ no grupo LC. Isto sugere uma redução da complacência, que é decorrente diretamente da lesão criogênica, mais importante que o comprometimento dessa variável observado no grupo BS, quando o balão subdural foi desinsuflado.

Aparentemente, a diferença entre os dois modelos estudados sugere que, no modelo de balão subdural, a complacência do encéfalo aproxima-se mais do ponto "b" da curva de pressão/volume no território intracraniano, como descrito, pela primeira vez ${ }^{8}$, em 1965 (Figura 6). Já no modelo de lesão criogênica extensa, a complacência parece estar mais próxima da região "c" da mesma curva, especialmente mais tardiamente. Isto sugere que, nos moldes das lesões estipuladas, o modelo de lesão criogênica seja mais prejudicial ao encéfalo e altere mais a complacência e o mecanismo de autorregulação vascular.

Embora durante o segundo teste de complacência (M4), a PIC não tenha mostrado diferença significativa entre os grupos ( $p=0,98)$, a evolução das duas formas de lesão encefálica mostrou tendências nitidamente distintas no transcorrer do tempo, ao longo da experimentação.

No grupo LC a formação de edema e a perda da ARVE pareceram progredir com o tempo, o que também sugere uma lesão mais grave, prolongada e irreversível (apoptose). Mesmo que o nível de hipertensão arterial induzida tenha sido algo maior no grupo BS que em LC, as repercussões sobre a PIC, e, portanto sobre a complacência do encéfalo, foram ainda maiores com a lesão criogênica extensa.

As alterações da PIC com modelos de lesão a frio nem sempre foram evidentes nos estudos anteriores ${ }^{11,12,14,15}$, provavelmente porque nesses modelos a extensão da lesão tenha sido pequena e insuficiente para comprometer significativamente os mecanismos de compensação do aumento da PIC, resultando em um modelo de encéfalo agredido, mas cuja extensão da lesão não tivesse sido suficiente para elevar a PIC, que se manteve praticamente inalterada em todos esses modelos.

Segundo os achados de um grupo de pesquisadores, no trauma cranioencefálico o edema cerebral é o componente mais importante na fisiopatologia deste tipo de agressão ${ }^{4}$. Assim, optou-se por realizar uma agressão que modificasse muito, não só a reatividade vascular, como outros modelos, mas também a permeabilidade da barreira hematoencefálica. Outros pesquisadores estudaram as variáveis hemodinâmicas e a PIC durante a lesão criogênica do encéfalo e determinaram que o volume da lesão está intimamente correlacionado às alterações do fluxo sangüíneo encefálico ${ }^{12}$.

A extensão da lesão criogênica escolhida nesse trabalho parece mostrar bem as alterações estudadas, entretanto, a literatura ainda é escassa e inconclusiva a respeito desse modelo, particularmente na quantificação do tempo de resfriamento do encéfalo, no ponto de contato direto com o nitrogênio líqüido, isto é, se no osso, na dura-máter ou no cérebro, e na extensão da superfície resfriada. Neste modelo optou-se por circunscrever uma pequena superfície da calota crania- 
na e expor ao $\mathrm{N}_{2}$ líqüido por um tempo maior do que o empregado por outros autores.

A temperatura timpânica, no grupo LC, sofreu redução significativa durante o estabelecimento da lesão criogênica em M2 ( $p=0,006)$, diferenciando-se da temperatura do grupo $B S(p=0,02)$, e mantendo-se assim até o final do experimento. Essa diminuição da temperatura deveu-se principalmente ao mecanismo de lesão, que é gerado pelo resfriamento intenso e da proximidade do sensor de temperatura.

Concluiu-se que o modelo experimental de lesão criogênica pode ser utilizado no estudo da complacência encefálica. Ele é comparável ao modelo de balão subdural do ponto de vista das variáveis da dinâmica encefálica, pelo menos no intervalo de tempo observado.

Concluiu-se, também, concordando com os achados de outro grupo ${ }^{12}$, que a extensão da lesão é fundamental na determinação do comprometimento ao mecanismo de ARVE, e ainda deva ser melhor padronizada em estudo posterior, com diferentes intensidades e tempos de resfriamento no modelo de lesão criogênica para que se possa estabelecer uma maior precisão na proporção entre a lesão e a redução da complacência resultante.

Alesão encefálica resultante do resfriamento com nitrogênio líqüido foi mensurada em dois momentos: a fresco e após fixação em formalina por duas semanas para estabelecimento de correlação entre o tempo de resfriamento e a extensão da lesão obtida. Todo o hemisfério encefálico lesado foi corado pela hematoxilina-eosina e analisado sob microscopia óptica durante o desenvolvimento de estudo piloto de outro trabalho da mesma linha de pesquisa que este, encontrando-se em fase de preparação para publicação. No modelo da lesão criogênica, particularmente se extensa, postulou-se que, pelo menos aparentemente, o aumento da PIC parece ser um processo gradual e evolutivo, e seus limites ainda carecem melhor esclarecimento.

A evolução fisiopatológica da lesão criogênica é bem distinta da lesão obtida pela insuflação de balão subdural, e nesta, talvez houvesse a necessidade de maior insuflação ou de mais tempo de hipertensão intracraniana. Entretanto, as repercussões associadas ao deslocamento de estruturas encefálicas, o que não é acompanhado por lesão de proporcional intensidade na barreira hematoencefálica, não representam bem as alterações observadas nas vítimas de traumatismo cranioencefálico grave, onde a contusão parenquimatosa predomina, como no modelo de lesão criogênica, apresentando lesão tissular mais intensa e grave da barreira hematoencefálica que os hematomas simulados pelo modelo do balão.

\section{Effects of Induced Hypertension on Brain Compliance and Perfusion Pressure in Experimental Intracranial Hypertension: Comparison between Cryogenic Brain Injury and Subdural Balloon}

Nelson Mizumoto TSA, M.D.; Humberto Katsuji Tango, TSA, M.D.; Marcelo Lacava Pagnocca TSA, M.D.

\section{INTRODUCTION}

Brain is an elastic organ which, in normal conditions, may mildly and rapidly change in volume by changing cerebrospinal fluid (CSF) and/or blood content, eventually changing intracranial pressure (ICP) depending on brain compliance $(B C)^{1,2}$. Brain compliance - also referred as elastance - may be understood as the measure of nervous tissue viscoelasticity, or stiffness. In mathematical terms, elastance represents volume variation observed in an elastic body in response to pressure changes, while compliance expresses the reverse ratio. In biological terms, the concept expresses the ability to compensate intracranial volume increase, and is especially useful in pathological situations ${ }^{1,3}$. According to Monro-Kellie's doctrine, when the volume of one of the brain component increases, other(s) should proportionally decrease, otherwise ICP will increase. Sustained ICP increase above $15 \mathrm{mmHg}$ is a pathological condition defined as intracranial hypertension $(\mathrm{ICH})^{2}$.

Traumatic brain injury (TBI), especially when severe, promotes $\mathrm{ICH}^{4}$ - originated from water (edema), CSF (hydrocephalus), intravascular blood (brain swelling) or extravascular blood (extradural, subdural or intraparenchymatous hematomas) - in addition to impairing brain vascular auto-regulation (BVAR) ${ }^{5}$ and may decrease perfusion pressure depending on mean blood pressure $(\mathrm{MBP})^{6}$. In addition, TBI patients suffer trauma-induced pain and also different manipulations during treatment, such as intubation and tracheal aspiration, skin punctures, vesical and nasogastric catheters, which are potentially nociceptive stimulations promoting MBP increase. This induced hypertension may increase blood volume in the intracranial space, especially if there is BVAR and BC decrease. For these reasons, TBI may change brain compliance and perfusion in different ways.

Type and intensity of those changes are dependent on the way, speed and intensity in which TBI promotes $\mathrm{ICH}$, so, the experimental model is major determinant of observed results and inferred conclusions.

Known experimental models try to mimic the same phenomena of neuronal aggression after TBI. Two specific models are highlighted for having specific characteristics similar to those found in THI patients: decreased brain compliance and perfusion pressure.

The first of these models is obtained with gradual inflation of a balloon located in the intracranial space (extradural, 
subdural or intraventricular). Major advantage is to increase ICP in a controllable way ${ }^{7}$, allowing for intensity to be maintained in desired times and values ${ }^{3}$, thus helping the study of brain compliance and perfusion. However, although being possible to reach $\mathrm{ICH}$ values to decrease $\mathrm{CPP}^{8}$, blood-brain barrier (BBB) injury is not so severe as compared to other models or to TBI in humans ${ }^{9}$, which is a disadvantage of this model.

The second model creates a focal brain injury by intensive cooling (cryogenic injury). Histopathological brain changes ${ }^{10}$ are observed in this model, such as BBB rupture, edema, intraparenchymatous hemorrhage and major impairment in BVAR mechanism ${ }^{5}$, promoting increased intravascular volume as a consequence of stasis ${ }^{8}$. However, the mass effect, as observed in the balloon model, is incipient.

In cryogenic injury models, BBB injury and resulting edema seem to be more important in decreasing $B C$ as compared to balloon models in which mass effect seems to be the primary component to decrease BVAR, thus changing BC.

Both models have been often used to study TBI without taking those differences into consideration and were still not compared to check changes in BVAR and BC, especially in the presence of hypertension concomitant to $\mathrm{ICH}$, so it is still not clear whether they are in fact comparable.

This study aimed at comparing the effect of induced hypertension on brain compliance and perfusion pressure in two experimental models in dogs: brain injury induced by subdural balloon inflation or by cryogenic cooling.

\section{METHODS}

This study involved 18 mixed breed dogs of both genders, weighing 10 to $20 \mathrm{~kg}$, in 10-hour fast, without preanesthetic medication and after evaluation and approval by the veterinary in charge of the experimental animals facility.

Dogs were placed in transparent acrylic boxes saturated and ventilated with $4 \%$ halothane in a $10 \mathrm{~L} \cdot \mathrm{min}^{-1} \mathrm{O}_{2} / \mathrm{N}_{2} \mathrm{O}(1: 1)$ gas flow. After unconsciousness and decreased respiratory rate to $12 \mathrm{rpm}$, animals were placed on Claude-Bernard device in the supine position and were intubated. Inspired halothane fraction was decreased to $2 \%$, mechanically controlled ventilation was installed with tidal volume of $8 \mathrm{~mL} . \mathrm{kg}^{-1}$, I/E ratio of $1: 1$ and frequency was adjusted to maintain expired $\mathrm{CO}_{2}$ in 33 $\pm 2 \mathrm{mmHg}$.

Pulse oximeter probe was placed on the tongue and ECG in $D_{\|}$lead on paws; tympanic thermometer was placed in right side, in addition to capnography and blood gases analyzer. Subcutaneous infiltration with $1 \mathrm{~mL}$ of $1 \%$ lidocaine was performed before every incision. Right femoral artery was dissected and catheterized for continuous mean blood pressure (MBP) monitoring and right femoral vein was dissected and catheterized for central venous pressure (CVP) monitoring. Left femoral artery was dissected and catheterized to collect the blood samples for biochemical dosages and ipsilateral femoral vein was dissected and catheterized for infusion of anesthetics and saline. Halothane inspired fraction was decreased to $1 \%$ and $2 \mu \mathrm{g} \cdot \mathrm{kg}^{-1}$ fentanyl and $0.08 \mathrm{mg} \cdot \mathrm{kg}^{-1}$ pancuronium were administered. Position was changed to "sphinx" position. Skullcap was surgically exposed. Circular trepanation with $1 \mathrm{~cm}$ diameter was performed $1.5 \mathrm{~cm}$ laterally to skull median line and intracerebral sensor was introduced to monitor ICP.

Animals were randomly distributed in two equal groups according to the scheme:

1 - Subdural balloon group (SB) - $3 \mathrm{~mm} \times 5 \mathrm{~mm}$ trepanation and dural opening in the hemisphere contralateral to ICP catheter, introduction of $2 \mathrm{~cm}$ of Foley's catheter distal edge in the subarachnoid space. Tight sealing with acrylic resin. Slow and gradual balloon inflation with $0.9 \%$ $\mathrm{NaCl}$ solution for maintenance ICP around $40 \mathrm{mmHg}$ during 20 minutes (Figures 1 and 2).

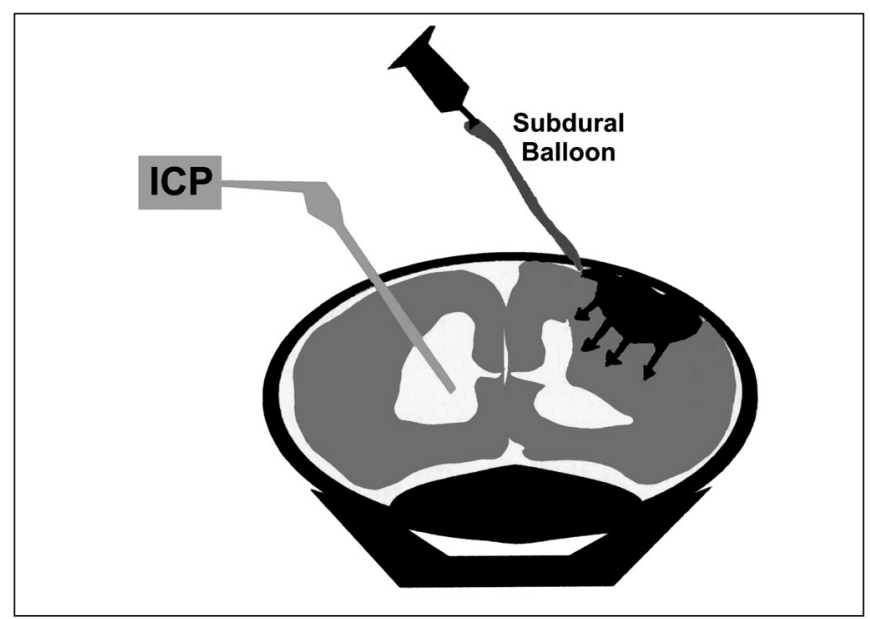

Figure 1 - Schematic Representation of Group SB

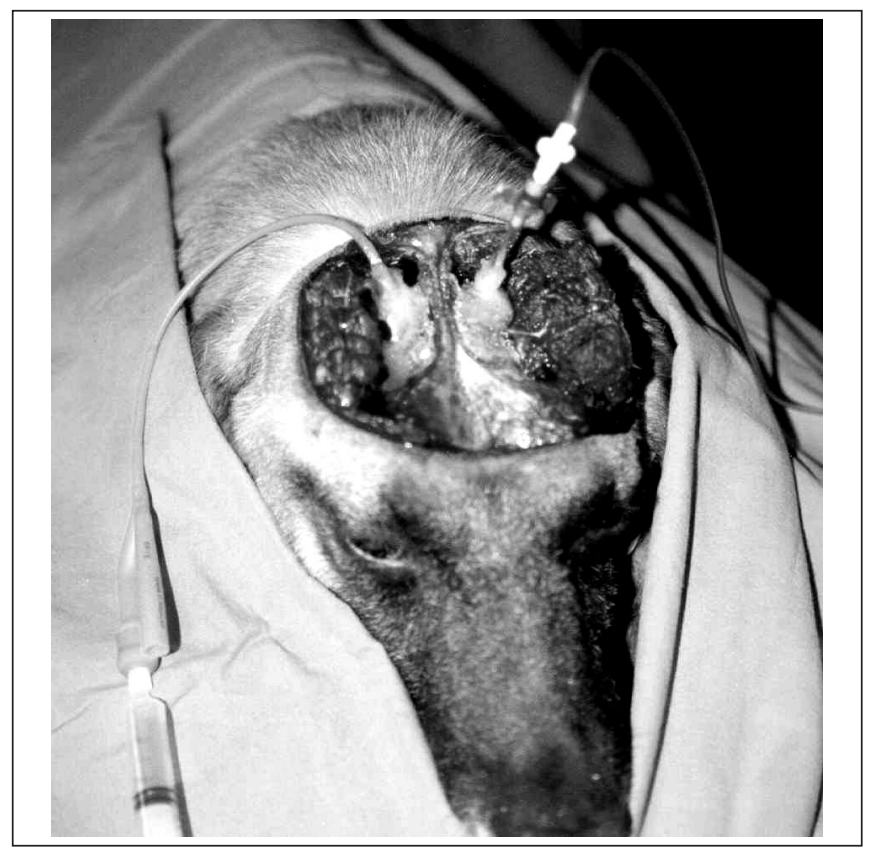

Figure 2 - Experimental Preparation of group SB 
2 - Cryogenic injury group $(\mathrm{Cl})$ - Plastic funnel fixation with acrylic resin to the skullcap contralateral to ICP trepanation. The area delimited by funnel edge fixed to skullcap was a $25 \mathrm{~mm}$ diameter circumference. Then liquid nitrogen was poured through the funnel on the bone during 20 minutes (Figures 3 and 4).

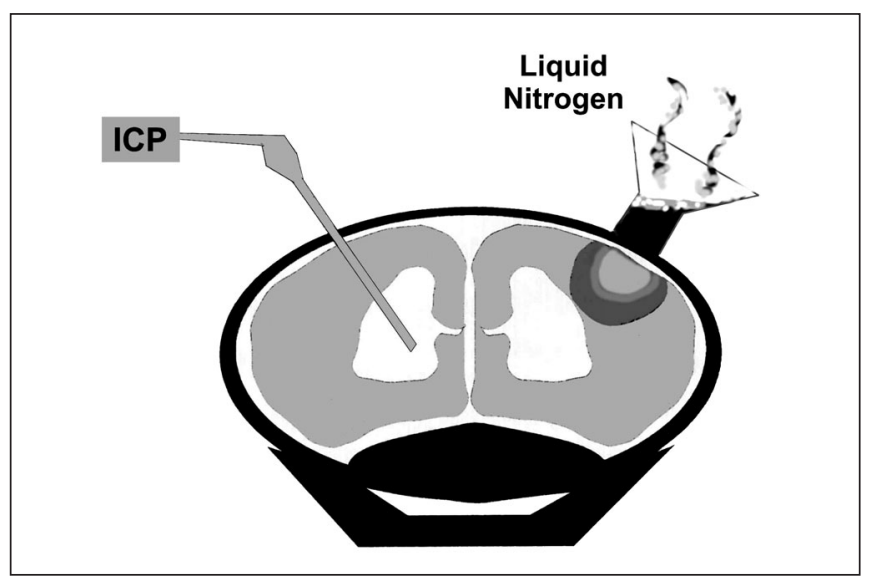

Figure 3 - Schematic Representation of Group Cl

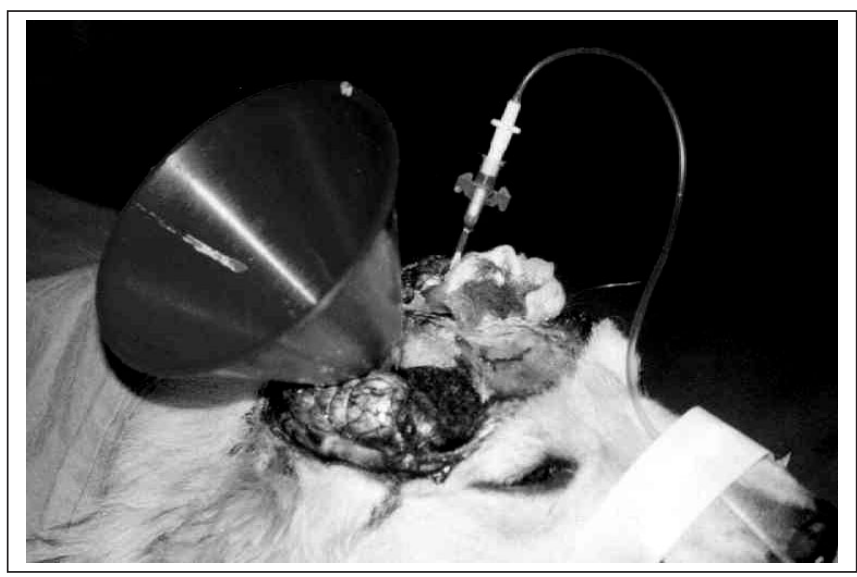

Figure 4 - Experimental Preparation of Group $\mathrm{Cl}$

Brain compliance test: is the core of the experimental model proposed in this study and depends on the functional status of brain vascular auto-regulatory mechanism. It consists on controlled MBP increase from a baseline value around 90 $\mathrm{mmHg}$ to the limit of $140 \mathrm{mmHg}$ and in measuring resulting ICP and CPP.

Blood brain volume (BBV) is a function of brain blood flow (BBF). So, increased blood pressure within these pressure limits barely changes intracranial volume if BVAR mechanism is intact, and when it is changed, it is to a mildly lower value; so, this induced hypertension does not increase ICP ${ }^{5}$. Conversely, if brain is aggressed and auto-regulation mechanism is functionally impaired ${ }^{5}$, then blood pressure increase within physiological blood pressure interval, will passively dilate resistance arterioles and increase BBF, and as a conse- quence, increase BBV ${ }^{8}$ and intracranial volume which ends up increasing ICP and decreasing brain compliance ${ }^{2}$.

As from this conclusion one may qualitatively infer the functional status of vascular auto-regulation ${ }^{16}$ and quantify $B C$ by MBP variation within the physiological interval of this phenomenon.

When test is negative, that is, when there is no significant ICP change, the assumption is that BVAR mechanism is intact (curve "a", Figure 5) and that parenchymal elasticity is within the linear region of the brain compliance curve (segment "a", Figure 6).

When the test is positive, that is, when there is significant ICP increase with increased MBP, the assumption is that there is functional BVAR impairment (curve "b", Figure 5) and that brain compliance is partially decreased during moderate $\mathrm{ICH}$ (segment "b") or significantly decreased in severe ICH (segment "c") of Langfitt's curve ${ }^{8}$ (Figure 6).

Possible BBV change is a function of BVAR activity, but when present, it also depends on the agnitude of pressure variation. If mean blood pressure variation is always the same and

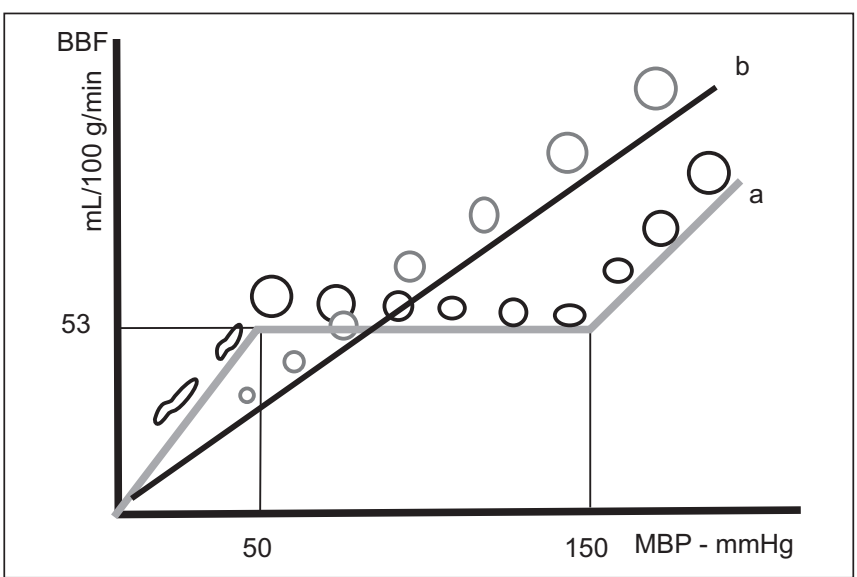

Figure 5 - Brain Blood Flow as a Function of Mean Blood Pressure. The circles represent the resistance vessels caliber

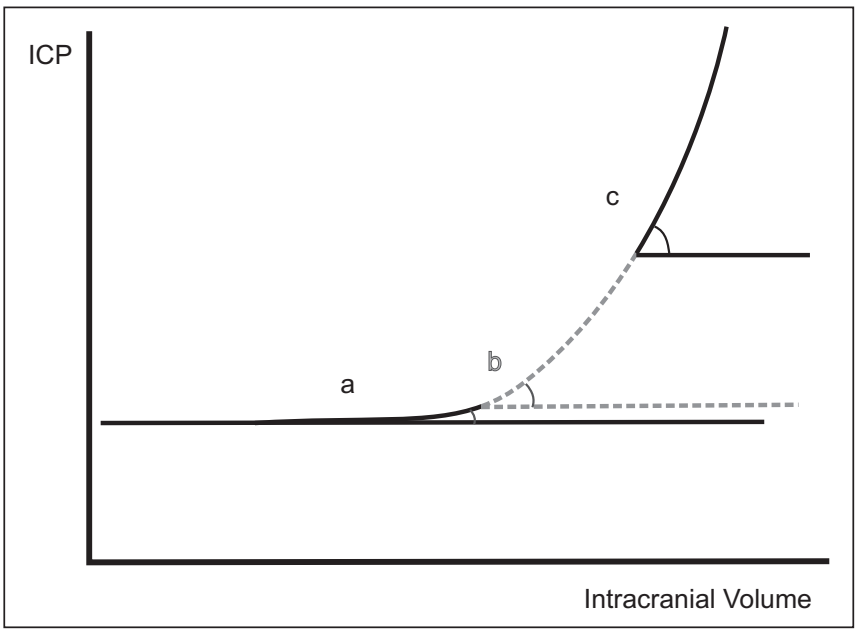

Figure 6 - Intracranial Pressure as a Function of Intracranial Volume

Revista Brasileira de Anestesiologia Vol. 55, № 3, Maio - Junho, 2005 
all other conditions interfering with brain blood flow are maintained constant, one may consider observed ICP increase as dependent only on BVAR functional status and level of brain compliance.

So, considering that: 1) MBP increases are arbitrary and defined by investigators, thus similar in different study moments; 2 ) induced hypertensions (final less initial MBP) end up generating brain blood flow increases also similar and proportional to the intensity of MBP variation; and 3) ICP increase is just a consequence of these blood volume increases within the skull, then a ratio may be established between ICP variation as a function of MBP variation, that is, an absolute pressure ratio in pressure terms.

MBP increase was arbitrarily determined as $50 \mathrm{mmHg}$ for being a sufficiently high value to increase ICP in case of BVAR incompetence and to be obtained through titrated and controlled infusion of a diluted norepinephrine solution. We started from a baseline value of approximately $90 \mathrm{mmHg}$ toward a ceiling of approximately $140 \mathrm{mmHg}$, being careful not to go beyond the upper auto-regulatory phenomenon limit. We have also tried to rigorously start from the same MBP baseline value and to always repeat each MBP increase within the same time interval.

Analyzed parameters - MBP, ICP and CPP - were collected in the following moments:

(M0) - Moment zero - after stabilization, corresponding to 20 minutes after sensors insertion. In this period, sodium (osmolarity), $\mathrm{pH}, \mathrm{PaCO}_{2}, \mathrm{PaO}_{2}, \mathrm{O}_{2}$ saturation (BVAR) and hematocrit (viscosity) of arterial blood were also evaluated to check whether groups were similar in these variables.

(M1) - Moment 1 - First brain compliance test: titrated norepinephrine infusion until target MBP and recording of resulting ICP and CPP - corresponding to normal brain response (BVAR and $B C$ ) to induced hypertension.

(M2) - Moment 2 - Brain injury was started 15 minutes after the end of norepinephrine infusion in M1, when hemodynamic variables had already returned to baseline values: subdural balloon inflation until ICP above $30 \mathrm{mmHg}$ in Group SB or beginning of cryogenic injury by pouring liquid nitrogen through the funnel during 20 minutes in group $\mathrm{Cl}$.

(M3) - Moment 3 - 20 minutes after subdural balloon inflation in SB group with lowest ICP of $30 \mathrm{mmHg}$, and 20 minutes after end of cryogenic injury, that is, 40 minutes after cryogenic injury beginning in group $\mathrm{Cl}$, corresponding to end of injuries establishment in both groups.

(M4) - Moment 4 - Second compliance test: titrated norepinephrine infusion until target MBP and recording of resulting ICP and CPP - corresponding to injured brain response (BVAR and $B C$ ) to induced hypertension. Figure 7 shows the chronology of the experiment.

Animals were sacrificed at the end of the experiment by increasing anesthetic depth followed by bolus $10 \mathrm{~mL}$ of $19.1 \%$ potassium chloride injection. Statistical analysis used paired

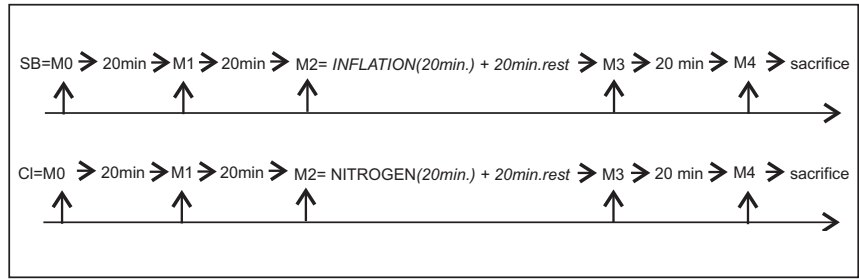

Figure 7 - Chronological Representation of Experimental Preparation

Student's $t$ test to compare variations within the same group between moments $\mathrm{M} 0$ and $\mathrm{M} 1$, with intact brain; Student's $t$ test for two different samples to compare both groups in moment M0 (normal pressure) and M1 (hypertension), to check whether animals belonged to the same sampling universe; Student's $t$ test for two different samples was used to compare variables between groups in the following moments:

1. M2 when balloon inflation was started to reach ICP between 30 and $50 \mathrm{mmHg}$ in the subdural balloon group and beginning of cooling in cryogenic injury group.

2. M3 20 minutes after balloon inflation in subdural balloon group and 40 minutes after cooling in cryogenic injury group.

3. M4 after second norepinephrine dose infusion to promote hypertension in both groups.

Paired Student's $t$ test was used to compare within group in the following moments:

1. Between M2 and M3 to check changes between these moments, because injury mechanisms were different for both groups.

2. Between M3 and M4 to check whether norepinephrine infusion had changed each specific type of injury.

\section{RESULTS}

Animals of both groups belonged to the same sampling universe when evaluated by hemodynamic parameters (mean blood pressure and heart rate), brain parameters (intracranial and perfusion pressure), metabolic parameters (sodium, potassium, partial $\mathrm{CO}_{2}$ arterial pressure, hematocrit and temperature) and morphometric parameters (weight) in M0 (Tables I, II and III).

Table I - Biochemical Variables $\left(\mathrm{Na}^{+}, \mathrm{K}^{+}\right.$, Hematocrit and Partial $\mathrm{CO}_{2}$ Arterial Pressure) and Morphometric Parameter (weight) for Groups SB and $\mathrm{Cl}$ in Moment M0

\begin{tabular}{lccc}
\hline Parameters & $\mathrm{SB}^{*}$ & $\mathrm{Cl}^{*}$ & $\mathrm{p}$ \\
\hline Weight $(\mathrm{kg})$ & $14.2 \pm 2.8$ & $12.5 \pm 1.7$ & 0.67 \\
$\mathrm{Na}+\left(\mathrm{mEq} \cdot \mathrm{L}^{-1}\right)$ & $145.2 \pm 1.5$ & $144.6 \pm 2.1$ & 0.81 \\
$\mathrm{~K}+\left(\mathrm{mEq}{ }^{\mathrm{L}-1}\right)$ & $3.8 \pm 0.3$ & $3.6 \pm 0.3$ & 0.04 \\
Hematocrit $(\%)$ & $37.8 \pm 3.9$ & $39.7 \pm 6.8$ & 0.54 \\
$\mathrm{PaCO}_{2}(\mathrm{mmHg})$ & $34.2 \pm 3.8$ & $34.3 \pm 3.5$ & 0.65 \\
\hline${ }^{*}$
\end{tabular}


Mean blood pressure (MBP): There has been MBP increase in $\mathrm{M} 1$ in groups $\mathrm{SB}(145.7 \pm 6.2 ; \mathrm{p}<0.05)$ and $\mathrm{Cl}(140.7 \pm 11.5$; $\mathrm{p}<0.05)$. In addition, intensity of induced hypertension (IH) was similar for both groups in M1 $(p=0.25)$, as desired. After rest and stabilization period, corresponding to beginning of injury in both groups, MBP in M2 has returned to baseline values for both groups. Similarly, MBP at rest until beginning of second compliance test in $\mathrm{M} 3$ was also similar for both groups ( $\mathrm{SB}=97 \pm 7.1$ and $\mathrm{Cl}=91.8 \pm 10.3 ; \mathrm{p}=0.31$ ). After establishing the injury in $\mathrm{M} 3, \mathrm{MBP}$ was similar to $\mathrm{M} 2$ for $\mathrm{SB}$ (101.3 \pm 8.1 ; $\mathrm{p}=0.65)$ and $\mathrm{Cl}(95.8 \pm 9.4 ; \mathrm{p}=0.41)$. As expected, there has been significant MBP increase during IH both for SB (148.1 \pm $10 ; p<0.001)$ and $\mathrm{Cl}(138.4 \pm 8 ; p<0.001)$. In this last test, MBP was slightly higher for SB as compared to $\mathrm{Cl}(p=0.04)$ (Table II and Figure 8).

Intracranial pressure (ICP): There has been significant ICP increase in $\mathrm{M} 1$ both for $\mathrm{SB}(9.5 \pm 4.7 ; \mathrm{p}=0.04)$ and $\mathrm{Cl}(11.3 \pm$ $3.7 ; p=0.01)$, however similar for both groups $(p=0.21)$. There has been significant ICP increase in M2 for SB (37 \pm $7.4 ; p=0.01$ ) with balloon inflation, while ICP was maintained unchanged for $\mathrm{Cl}(12 \pm 3.7 ; \mathrm{p}=0.18)$. At this moment, ICP values became different $(p<0.001)$. In M3, ICP was maintained high in SB group, with no difference as compared to M2 (32 \pm 12.9 and $p=0.39$ ). In group $\mathrm{Cl}, 20$ minutes after cryogenic injury, or M3, ICP has significantly increased (26 \pm 6.6 ; $p$ $<0.001)$, again making ICPs similar $(p=0.39)$. In M4, during second compliance test, group SB increase was insignificant $(38 \pm 17.1 ; p=0.12)$ however group $\mathrm{Cl}$ increase was significant $(35.7 \pm 6.7 ; p<0.001)$, although ICP values were similar for both groups ( $p>0.98$ (Table III and Figure 9).

Brain perfusion pressure (BPP): There has been significant CPP increase between $\mathrm{M} 0$ and $\mathrm{M} 1$, both for SB $(136 \pm 6.9 ; \mathrm{p}<$ $0.001)$ and $\mathrm{Cl}(126 \pm 5 ; \mathrm{p}<0.001)$, however higher for group

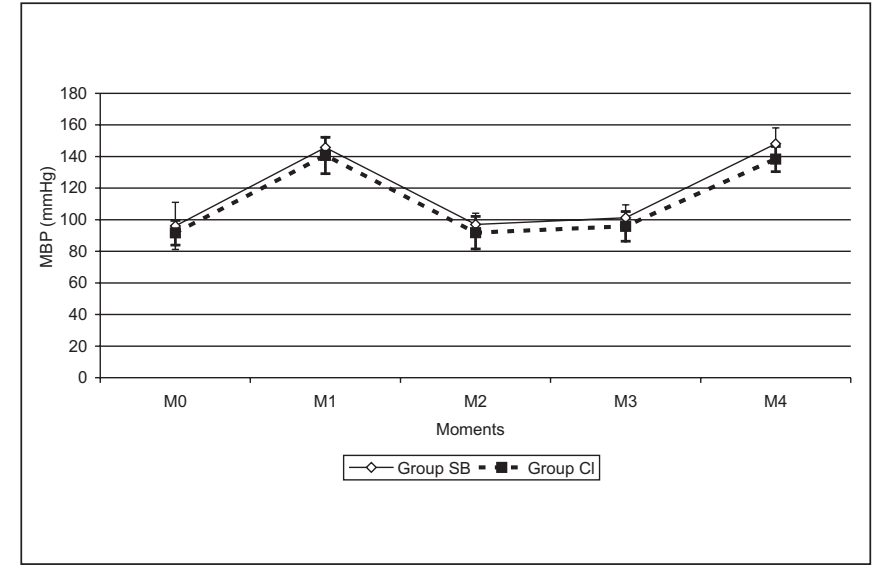

Figure 8 - Mean Blood Pressure of Groups SB and CI in Moments $\mathrm{M} 0, \mathrm{M} 1, \mathrm{M} 2, \mathrm{M} 3$ and $\mathrm{M} 4$

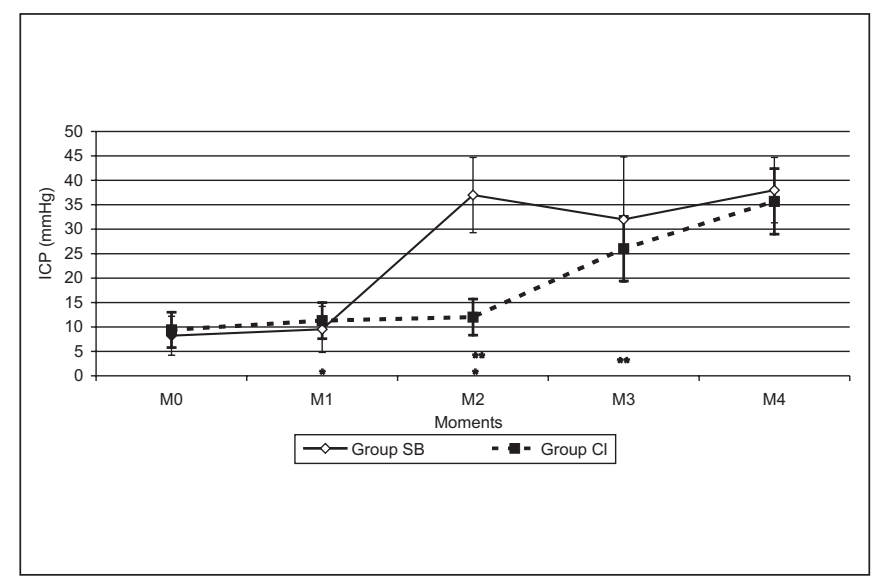

Figure 9 - Intracranial Pressure (ICP) in Groups SB and $\mathrm{Cl}$ in $\mathrm{Mo}-$ ments M0, M1, M2, M3 and M4

Table II - Mean Blood Pressure (MBP) for Groups SB and Cl in Moments M0, M1, M2, M3 and M4

\begin{tabular}{|c|c|c|c|c|c|c|}
\hline Moments & $\begin{array}{c}\text { SB } \\
\text { Mean } \pm \text { SD }\end{array}$ & $\begin{array}{c}\mathrm{Cl} \\
\text { Mean } \pm \mathrm{SD}\end{array}$ & $\begin{array}{c}\mathrm{SB} \times \mathrm{Cl} \\
\text { Significance }\end{array}$ & Interval & $\begin{array}{c}\text { SB X SB } \\
\text { Significance }\end{array}$ & $\begin{array}{c}\mathrm{Cl} \times \mathrm{Cl} \\
\text { Significance }\end{array}$ \\
\hline M0 & $96.1 \pm 14.9$ & $91.7 \pm 7.8$ & 0.32 & & & \\
\hline M1 & $145.7 \pm 6.2$ & $140.7 \pm 11.5$ & 0.25 & M0 - M1 & $<0.001$ & $<0.001$ \\
\hline M2 & $97 \pm 7.1$ & $91.8 \pm 10.3$ & 0.31 & M1 - M2 & $<0.001$ & $<0.001$ \\
\hline M3 & $101.3 \pm 8.1$ & $95.8 \pm 9.4$ & 0.19 & M2 - M3 & 0.06 & 0.41 \\
\hline M4 & $148.1 \pm 10$ & $138.4 \pm 8$ & 0.04 & M3 - M4 & $<0.001$ & $<0.001$ \\
\hline
\end{tabular}

Values in $\mathrm{mmHg}$ expressed in Mean $\pm \mathrm{SD} ; \mathrm{SB}=$ Sudural balloon; $\mathrm{Cl}=$ Cryogenic injury

Table III - Intracranial Pressure (ICP) of Groups SB and Cl in Moments M0, M1, M2, M3 and M4

\begin{tabular}{|c|c|c|c|c|c|c|}
\hline Moments & $\begin{array}{c}\text { SB } \\
\text { Mean } \pm S D\end{array}$ & $\begin{array}{c}\mathrm{Cl} \\
\text { Mean } \pm \mathrm{SD}\end{array}$ & $\begin{array}{c}\mathrm{SB} \times \mathrm{Cl} \\
\text { Significance }\end{array}$ & Interval & $\begin{array}{c}\text { SB X SB } \\
\text { Significance }\end{array}$ & $\begin{array}{c}\mathrm{Cl} \times \mathrm{Cl} \\
\text { Significance }\end{array}$ \\
\hline MO & $8.2 \pm 4$ & $9.4 \pm 3.6$ & 0.27 & & & \\
\hline M1 & $9.5 \pm 4.7$ & $11.3 \pm 3.7$ & 0.21 & M0 - M1 & 0.05 & 0.01 \\
\hline M2 & $37 \pm 7.7$ & $12 \pm 3.7$ & $<0.001$ & $\mathrm{M} 1$ - M2 & $<0.001$ & 0.18 \\
\hline M3 & $32 \pm 12.8$ & $26 \pm 6.6$ & 0.39 & $\mathrm{M} 2-\mathrm{M} 3$ & 0.36 & $<0.001$ \\
\hline M4 & $38 \pm 6.7$ & $35.7 \pm 6.7$ & 0.98 & M3 - M4 & 0.12 & $<0.001$ \\
\hline
\end{tabular}

Values in $\mathrm{mmHg}$ expressed in Mean $\pm \mathrm{SD}$; $\mathrm{SB}=$ Subdural balloon; $\mathrm{Cl}=$ Cryogenic injury 
EFFECTS OF INDUCED HYPERTENSION ON BRAIN COMPLIANCE AND PERFUSION PRESSURE IN EXPERIMENTAL INTRACRANIAL HYPERTENSION: COMPARISON BETWEEN CRYOGENIC BRAIN INJURY AND SUBDURAL BALLOON

Table IV - Brain Perfusion Pressure (CPP) in Groups SB and Cl in Moments M0, M1, M2, M3 and M4

\begin{tabular}{|c|c|c|c|c|c|c|}
\hline Moments & $\begin{array}{c}\text { SB } \\
\text { Mean } \pm \text { SD }\end{array}$ & $\begin{array}{c}\mathrm{Cl} \\
\text { Mean } \pm \mathrm{SD}\end{array}$ & $\begin{array}{c}\mathrm{SB} \times \mathrm{Cl} \\
\text { Significance }\end{array}$ & Interval & $\begin{array}{c}\text { SB X SB } \\
\text { Significance }\end{array}$ & $\begin{array}{c}\mathrm{Cl} \times \mathrm{Cl} \\
\text { Significance }\end{array}$ \\
\hline MO & $87.8 \pm 16.5$ & $82.3 \pm 6.5$ & 0.19 & & & \\
\hline M1 & $136 \pm 6.9$ & $126 \pm 5$ & 0.002 & M0 - M1 & $<0.001$ & 0.02 \\
\hline M2 & $60 \pm 6.9$ & $79.8 \pm 10.2$ & $<0.001$ & M1 - M2 & $<0.001$ & $<0.001$ \\
\hline M3 & $72.6 \pm 13$ & $69.8 \pm 9.9$ & 0.43 & M2 - M3 & 0.03 & 0.01 \\
\hline M4 & $110.1 \pm 17.1$ & $102.6 \pm 8.3$ & 0.16 & M3 - M4 & $<0.001$ & $<0.001$ \\
\hline
\end{tabular}

Values in $\mathrm{mmHg}$ expressed in Mean $\pm \mathrm{SD}$; $\mathrm{SB}=$ Subdural balloon; $\mathrm{Cl}=$ Cryogenic injury

Table V - Tympanic Temperature in Groups SB and Cl in Moments M0, M1, M2, M3 and M4

\begin{tabular}{|c|c|c|c|c|c|c|}
\hline Moments & $\begin{array}{c}\text { SB } \\
\text { Mean } \pm \text { SD }\end{array}$ & $\begin{array}{c}\mathrm{Cl} \\
\text { Mean } \pm \mathrm{SD}\end{array}$ & $\begin{array}{c}\mathrm{SB} \times \mathrm{Cl} \\
\text { Significance }\end{array}$ & Interval & $\begin{array}{l}\text { SB X SB } \\
\text { Significance }\end{array}$ & $\begin{array}{c}\mathrm{Cl} \times \mathrm{Cl} \\
\text { Significance }\end{array}$ \\
\hline MO & $35.2 \pm 1.7$ & $34.5 \pm 1.2$ & 0.17 & & & \\
\hline M1 & $35.3 \pm 1.7$ & $34.5 \pm 1.3$ & 0.16 & M0 - M1 & 0.12 & 0.86 \\
\hline M2 & $34.5 \pm 1.8$ & $33.5 \pm 1.4$ & 0.02 & $\mathrm{M} 1-\mathrm{M} 2$ & 0.05 & 0.006 \\
\hline M3 & $35.2 \pm 1.9$ & $33.4 \pm 1.7$ & 0.04 & $\mathrm{M} 2-\mathrm{M} 3$ & 0.38 & 0.81 \\
\hline M4 & $35.3 \pm 1.7$ & $33.5 \pm 1.6$ & 0.03 & M3 - M4 & 0.66 & 0.02 \\
\hline
\end{tabular}

$\mathrm{SB}=$ Subdural balloon; $\mathrm{Cl}=$ Cryogenic injury

SB $(p=0.002)$. There has been CPP decrease in M2 for SB $(60 \pm 6.9 ; p<0.001)$ and $\mathrm{Cl}(79.8 \pm 10.2 ; p<0.001)$. CPP decrease for SB was higher as compared to $\mathrm{Cl}(\mathrm{p}<0.001)$. In $M 3$, since there has been CPP increase for $S B(72.6 \pm 13 ; p=$ $0.02)$ and decrease for $\mathrm{Cl}(69.8 \pm 9.9 ; \mathrm{p}=0.01) \mathrm{CPP}$ has become similar for both groups $(p=0.43)$. With IH in M4, CPP has increased for SB $(110.1 \pm 17.1 ; p<0.001)$ and $\mathrm{Cl}(102.6 \pm$ $8.3 ; p<0.001)$. This increase was similar for both groups $(p=$ $0.16)$ (Table IV and Figure 10).

Temperature: Tympanic temperature was significantly decreased in $\mathrm{M} 2$ for both groups: SB $(35.4 \pm 1.8 ; p=0.05)$ and $\mathrm{Cl}$ $(33.5 \pm 1.4 ; p=0.006)$, being lower for $\mathrm{Cl}(p=0.02)$ and remaining as such until M4 (Table V).

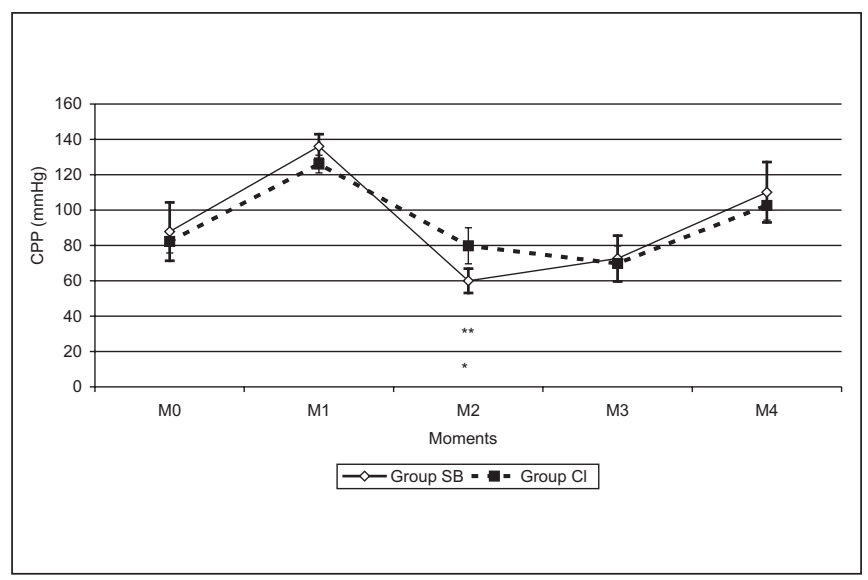

Figure 10 - Brain Perfusion Pressure (CPP) in Groups SB and $\mathrm{Cl}$ in Moments M0, M1, M2, M3 and M4

Revista Brasileira de Anestesiologia

Vol. 55, № 3, Maio - Junho, 2005

\section{DISCUSSION}

Different controlled brain aggression models have been used to study changes during and after traumatic brain injury $9,20,29,31-35$. Intuitively, the closest model to this type of aggression is skull contusion with solid objects.

However, this aggression often generates intracranial hematomas and bone fractures, which have unpredictable effects on brain compliance ${ }^{17}$. So, animal models allowing gradual and controlled ICP increase, thus more predictable, are the most widely used.

Subdural or epidural balloon inflation, intraventricular fluid infusion or other intracranial volume expansion methods may be more predictable as to value and time one wishes to maintain intracranial pressure. However, they do not severely affect blood-brain barrier patency and, as a consequence, nervous tissue edema does not seem to be an important factor in these models. Some authors ${ }^{18}$ suggest that after TBI, brain edema contribute to increase intracranial volume more than blood stasis caused by vasoparesis, that is, that ICP increase could be more a consequence of water volume increase, be it extravascular or intracellular, than of intravascular blood. In addition, ICP in severe traumatic brain injury is increased to a critical value enough to impair brain perfusion ${ }^{6}$. This extremely high intracranial pressure has not always been controlled in ICP studies ${ }^{17}$, which not always had brain perfusion pressure decreased to these critical levels to the point of impairing vascular reactivity and brain blood flow.

In this model, we have tried to maintain ICP above $30 \mathrm{mmHg}$ for group SB, as seen during severe traumatic brain injuries, and this has significantly decreased brain perfusion pressure from mean baseline value of $87 \mathrm{mmHg}$ in $\mathrm{M0}$ to $60 \mathrm{mmHg}$ in M2 ( $p$ < 0.001). With severe enough intracranial hypertension, in addition to decreasing perfusion, there is also muscle tone decrease of brain arterioles and major functional impair- 
ment of auto-regulatory mechanism, leading to passive resistance vascular bed dilatation and systemic hypertension ${ }^{8}$, further worsening pre-existing intracranial hypertension. A group ${ }^{6}$ has shown than when brain perfusion pressure is below $60 \mathrm{mmHg}$, brain blood flow starts to decrease. On the other hand, other authors ${ }^{16}$ have shown in anesthetized dogs that if blood pressure limits are maintained between 50 and $150 \mathrm{mmHg}$, brain compliance is directly proportional to perfusion pressure in normal conditions, that is with intact brain and preserved auto-regulation; and is indirectly proportional when brain is aggressed and its auto-regulatory mechanism is functionally impaired. With normal auto-regulation, mean blood pressure variation within physiological intervals is unable to significantly change BBF, and as a consequence to significantly change brain blood volume (BBV) and compliance. As opposed, with abolished auto-regulatory mechanism, BBV is increased due to increased BBF when MBP is increased, thus decreasing compliance.

Brain compliance evaluation by non-volumetric means, that is, without changing intracranial volume, has been used in different studies 19,29,30. These authors have studied ICP curve's B wave morphology in the presence of $\mathrm{IH}$ but have not found significant correlation between morphological changes in this wave and brain compliance evaluated by volumetric methods. They have also suggested that the best evaluation method would be volume/pressure ratio used by other authors ${ }^{2,20}$

During transportation, surgery or ICU stay, patients are frequently submitted to nociceptive stimulations increasing blood pressure to undesirable levels, what ends up decreasing neuronal perfusion exactly when brain vascular reactivity mechanism is functionally impaired.

Trying to reproduce this situation, our study has evaluated brain compliance exactly by means of induced hypertension, which also increases intracranial volume when brain is injured $^{6,8}$

Group SB intracranial hypertension was limited to values below $50 \mathrm{mmHg}$ to evaluate compliance in moments following the establishment of the injury.

The comparison of compliance between balloon and cryogenic injury models was important, since we did not know how this model would behave with a previously non-described injury extension.

In addition, excessive subdural balloon inflation could shift adjacent structures leading to menyngeal hemorrhages, hernias, venous system engorgement and hydrocephalus by CSF drainage obstruction ${ }^{1}$.

Cryogenic injury model has been used to evaluate blood-brain barrier patency both by gravimetric density and water volume in pathological exams of the aggressed region 10,14. Cryogenic injury also changes brain vascular reactivity in the involved area ${ }^{5}$. A group has shown that brain edema and swelling are significant already in the first 15 to $30 \mathrm{~min}$ utes after cryogenic injury ${ }^{10}$. However, most cryogenic injury experiments have not produced tissue damage extensive enough to increase $\mathrm{ICH}$ to values impairing brain perfusion pressure or causing enough damage to BVAR mechanism, 304 because either cooled surface was small or length of cryogenic agent contact with brain was too short ${ }^{11-15}$. Historically, cryogenic injury has not been used to study ICP $11,12,14$ because it seems that the extension of the injury described by these authors had not enough volume to significantly impair ICP and BVAR mechanism. Since the impaired volume described is not high, it seems that brain compliance compensation mechanisms, CSF and blood shifts, are effective in maintaining ICP within normal values ${ }^{8,12}$.

Our proposal was to evaluate brain compliance using two different models, however for both the level of proposed aggression was higher than what is normally studied and closer to those observed in severe traumatic brain injury patients. $\operatorname{MBP}(p=0.31), \operatorname{ICP}(p=0.27)$ and $\operatorname{CPP}(p=0.19)$ were similar for both groups in $\mathrm{M} 0$, confirming that they belonged to the same sampling universe. Other variables which could have also influenced brain blood flow ${ }^{36}$ and, as a consequence, results - central temperature (neuronal metabolism, $p=0.17$ ), plasma sodium (edemas of osmotic origin, $p=0.81$ ), hematocrit (blood viscosity, $\mathrm{p}=0.65$ ) and partial $\mathrm{CO}_{2}$ arterial pressure (changes in auto-regulatory mechanism, $p=0.37$ ) were also evaluated. Similarly, there were no significant differences between groups.

Special care was taken not to increase blood pressure above upper BVAR level (>150 $\mathrm{mmHg}$ ), to prevent hypertension of acting on BVAR per se ${ }^{21}$, or increasing capillary patency of normal brain ${ }^{21-23}$.

Anesthesia was maintained in adequate depth, however not excessive, with halogenate agent expired fraction below $1 \%$ (below DE95) due to the association of intravenous opioids in equal doses for both groups. We have also prevented hypercarbia to prevent cardiac arrhythmias and brain vessel dilatation with consequent intracranial blood volume increase during the infusion of norepinephrine associated to halothane ${ }^{24,25}$.

Our study has shown ICP threshold increase in significantly in $\mathrm{M} 1$ for both groups ( $p=0.05$ for $\mathrm{SB}$ and $\mathrm{p}=0.01$ for $\mathrm{Cl}$ ). This increase, however, was mild and fugacious, without corresponding biological meaning. Equally important is that there were no significant differences between groups in intensity of this change $(p=0,21)$. This brief ICP increase during induced hypertension was considered normal. Mild and temporary increase in ICP in response to hypertension in normal brain has also been observed by other investigators ${ }^{26,31}$.

Perfusion pressure in M1 has increased more in group SB as compared to group $\mathrm{Cl}(p=0.001)$, probably because there has been higher blood pressure increase concomitant with lower intensity increase of ICP in group SB as compared to group $\mathrm{Cl}$.

There has been sudden ICP increase for group SB in M2 ( $p<$ $0.001)$ and brain perfusion pressure decrease $(p<0.001)$ as we had proposed, and these values were maintained for 20 minutes with additional subdural balloon inflation whenever ICP tended to decrease, to obtain a certain level of brain vascular reactivity impairment. After this aggression period and decreased perfusion pressure, there has been mild, although

Revista Brasileira de Anestesiologia Vol. 55, N 3, Maio - Junho, 2005 
not significant blood pressure increase in M3 for both groups $(p=0.19)$.

Increased blood pressure at rest for both groups may be explained as central nervous system response to ischemia generated by intracranial hypertension ${ }^{27,28}$

There has been no ICP decrease for group SB in M2 and M3 ( $p$ $=0.36$ ). Although not significant, mild ICP decrease was probably due to compliance compensation mechanisms, CSF and blood shift to outside the skull while subdural balloon was deflated.

Although not representing significant differences when separately considered, increased blood pressure associated to decreased ICP has resulted in major brain perfusion pressure increase in M3 for group SB ( $p=0.03)$.

Significant mean blood pressure increase in M4 $(p<0.001$ for $\mathrm{SB}$ and $\mathrm{Cl}$ ) was expected because the effect of induced hypertension on aggressed brain ICP was exactly the objective of our study.

At this moment, in SB, although ICP increase has been observed, this increase was not significant $(p=0.12)$ as compared to previous moment M3 when blood pressure was normal for this same group. The result was increased brain perfusion pressure $(p<0.001)$ with norepinephrine infusion.

Maybe a more severe brain aggression was needed in group SB for further BVAR impairment. Time during which balloon has remained inflated could have been longer than 20 minutes or level of intracranial hypertension could have been above 50 $\mathrm{mmHg}$.

On the other hand, 20-minute ischemia seems to have been reasonable and balloon inflation maintaining ICP above 50 $\mathrm{mmHg}$ could shift adjacent brain structures and change blood and CSF dynamics.

On the other hand, in M2, even when liquid nitrogen injury had been already started, ICP was still unchanged for group $\mathrm{CI}$ ( $p$ $=0.18$ ), probably because changes in blood-brain barrier and brain vascular reactivity were not yet installed. Two authors have shown that both edema and brain swelling are manifested after brain cryogenic injury as early as 15 to 20 minutes after establishment of the injury and continue to evolve up to 12 hours after beginning of injured tissue reperfusion ${ }^{10}$.

Since at this moment MBP was similar to baseline value $(p=$ 0.93 ) and was also not different from group SB MBP ( $p=$ 0.31 ), CPP for group $\mathrm{Cl}$ was higher as compared to group SB $(p<0.001)$.

In moment $\mathrm{M} 3,40$ minutes after beginning of cryogenic injury, corresponding to 20 minutes of injury plus 20 minutes of rest for overcooled brain thawing and reperfusion, ICP started to increase $(p<0.001)$. At this moment, cryogenic injury repercussions on ICP and on brain vascular reactive mechanism were already present, in agreement to these authors' findings ${ }^{10}$.

With this ICP increase, CPP was decreased $(p=0.01)$ in this group $(\mathrm{Cl})$. In the same moment M3, CPP was similar for both groups $(p=0.43)$. In $M 4$, norepinephrine infusion has increased $\operatorname{MBP}(p<0.001)$, leading to CPP increase $(p<0.001)$ but also to ICP increase $(p<0.001)$ for group $C$. This suggests that decreased compliance, which is a direct conse- quence of cryogenic injury, was more important than the impairment of this variable observed in group SB when subdural balloon was deflated.

The difference between both studied models suggests that in the subdural balloon model brain compliance is closer to point "b" of intracranial pressure/volume curve, as described for the first time in $1965^{8}$ (Figure 6). In the extensive cryogenic injury model, however, compliance seems to be closer to region "c" of the same curve, especially more lately. This suggests that, in the conditions of our study, cryogenic injury model would be more prejudicial to brain and would further change compliance and vascular auto-regulation mechanism.

Although there were no significant ICP differences between groups during second compliance test (M4) $(p=0.98)$, the evolution of both brain injuries has shown clearly different trends along time throughout the experiment.

In group $\mathrm{Cl}$, edema and loss of BVAR seemed to progress with time, what also suggests a more severe, prolonged and irreversible injury (apoptosis). Although induced hypertension was somewhat higher for group SB as compared to $\mathrm{Cl}$, repercussions on ICP, thus on brain compliance, where even higher with extensive cryogenic injury.

ICP changes with cold injury models were not always apparent in previous studies ${ }^{11,12,14,15}$, probably because in these models, injury has been small and not enough to significantly impair increased ICP compensation mechanisms, resulting in a model of aggressed brain but with injury not large enough to increase ICP, which was maintained virtually unchanged in all those models.

According to some investigators, brain edema during traumatic head injury is the most important component of the pathophysiology of this type of aggression ${ }^{4}$. So, we decided for an aggression that would largely change not only vascular reactivity, as in other models, but also blood-brain barrier patency.

Other investigators have studied hemodynamic variables and ICP during brain cryogenic injury and have determined that injury volume is closely related to brain blood flow changes $^{12}$.

Cryogenic injury extension of this study seems to clearly show studied changes, however literature is still scarce and inconclusive about this model, especially in quantifying brain cooling time, in determining direct contact point with liquid nitrogen, that is, whether bone, dura or brain, and in determining the extension of cooled surface. We decided to circumscribe a small skullcap surface and expose it to liquid $\mathrm{N}_{2}$ for a longer time as compared to other authors. Tympanic temperature for group $\mathrm{Cl}$ was significantly decreased during cryogenic injury establishment in $M 2(p=0.006)$, being different from group SB temperature $(p=0.02)$ and remaining as such until the end of the experiment. This temperature decrease was especially due to proximity to thermal sensor and injury mechanism, wich is intensive cooling.

The conclusion was that experimental cryogenic injury model could be used to study brain compliance. It is comparable to subdural balloon model in terms of brain dynamic variables, at least during the time interval of our study.

We have also concluded, in agreement with other findings ${ }^{12}$, that injury extension is critical to determine BVAR mecha- 
nism impairment and that it should be better standardized in further studies, with different cooling intensities and times in the cryogenic injury model to establish the ratio between injury and resulting compliance decrease.

Brain lesion by liquid nitrogen cooling was measured in two moments: fresh and after fixation with formalin for two weeks to establish correlation between cooling time and lesion extension. The whole injured brain hemisphere was stained with hematoxylin-eosin and analyzed under optic microscopy during the development of pilot of a different study in the same research line of our group, which is being prepared for publication.

For cryogenic injuries, especially if extensive, it has been advocated that, seemingly at least, increased ICP seems to be a gradual and evolving process and that its limits are still lacking better explanation.

Pathophysiological evolution of cryogenic lesion is clearly different from subdural balloon inflation injury, where maybe more inflation or longer intracranial hypertension would be needed. However, repercussions related to brain structures shift, which is not followed by proportionally severe blood-brain barrier injury, do not well represent changes observed in severe traumatic head injury patients, where parenchymatous contusion predominates, as in the cryogenic injury model, presenting more intensive and severe tissue injury of blood-brain barrier as compared to hematomas simulated by the balloon model.

\section{REFERÊNCIAS - REFERENCES}

01. Johnston IH, Rowan JO - Raised intracranial pressure and cerebral blood flow: 3 . Venous outflow tract pressures and vascular resistances in experimental intracranial hypertension. $\mathrm{J}$ Neurol Neurosurg Psychiatry, 1974;37:392-402.

02. Miller JD - Intracranial volume-pressure relationships in pathological conditions. J Neurosurg, 1976;20:203-213.

03. Leech P, Miller JD - Intracranial volume-pressure relationship during experimental brain compression in primates. 1. Pressure responses to changes in ventricular volume. J Neurol Neurosurg Psychiatry, 1974;37:1093-1098.

04. Marmarou A, Barzo P, Fotouros P et al - Traumatic brain swelling in head injured patients: brain edema or vascular engorgement? Acta Neurochir, 1997;(Suppl70):68-70.

05. Reilly PL, Farrar JK, Miller JD - Vascular reactivity in the primate brain after acute cryogenic injury. J Neurol Neurosurg Psychiatry, 1977;40:1092-1101.

06. Wagner EM, Traystman RJ - Hidrostatic determinants of cerebral perfusion. Crit Care Med, 1986;14:484-490

07. Mizumoto $\mathrm{N}$ - Efeito do $\mathrm{NaCl} 7,5 \%$ nas pressões arterial e intracraniana em cães em choque hermorrágico, com e sem hipertensão intracraniana grave. Tese de doutorado, Disciplina Anestesiologia FMUSP, 1996.

08. Langfitt TW, Weinstein JD, Kassell NF - Cerebral vasomotor paralysis produced by intracranial hypertension. Neurology, 1965;15:622-641.

09. Clubb RJ, Maxwell RE, Chou SN - Experimental brain injury in the dog. The pharmacological effects of pentobarbital and sodium nitroprusside. J Neurosurg, 1980;52:189-196.
10. Schneider GH, Hennig S - Dynamics of posttraumatic brain swelling following a cryogenic injury in rats. Acta Neurochir, 1994;(Suppl60):437-439

11. Shackford SR, Zhuang J, Schmoker J - Intravenous fluid tonicity: effect on intracranial pressure, cerebral blood flow, and cerebral oxygen delivery in focal brain injury. J Neurosurg, 1992;76: 91-98.

12. Schmoker JD, Zhuang J, Shackford SR et al - Effect of lesion volume on cerebral hemodynamics after focal brain injury and shock. J Trauma, 1993;35:627-636.

13. Kaieda R, Todd MM, Warner DS - Prolonged reduction in colloid oncotic pressure does not increase brain edema following cryogenic injury in rabbits. Anesthesiology, 1989;71:554-560.

14. Albright AL, Latchaw RE, Robinson AG - Intracranial and systemic effects of hetastarch in experimental cerebral edema. Crit Care Med, 1984;12:496-500.

15. Trevisani GT, Shackford SR, Zhuang J et al - Brain edema formation after injury, shock, and resuscitation: effects of venous and arterial pressure. J Trauma, 1994;37:452-458.

16. Gray WJ, Rosner MJ - Pressure-volume index as a function of cerebral perfusion pressure. Part 2: The effects of low cerebral perfusion pressure an autoregulation. J Neurosurg, 1987;67: 377-380.

17. Engelborghs K, Verlooy J, Van Deuren B et al - Intracranial pressure in a modified experimental model of closed injury. Acta Neurochir, 1997;(Suppl70):123-125.

18. Marmarou $A$, Nakamura $T$, Sakamoto $\mathrm{H}$ et al - Development of Brain Edema following Fluid Percussion Injury, em: Inaba $Y$, Klatzo I, Spatz M - Brain Edema - Proceedings of the Sixth International Symposium, Berlin, Springer-Verlag, 1985;88-91.

19. Wilkinson HA, Schuman N, Ruggiero J- Nonvolumetric methods of detecting impaired intracranial compliance or reactivity. J Neurosurg, 1979;50:758-767.

20. Lecch P, Miller D - Intracranial volume-pressure relanshionships during experimental brain compression in primates. Effect of mannitol and hyperventilation. J Neurol Neurosurg Psych, 1974;37:1105-1111.

21. MacKenzie ET, Strandgaard S, Graham DI et al - Effects of acutely induced hypertension in cats on pial arteriolar caliber, local cerebral blood flow, and the blood-brain barrier. Circ Res, 1976;39:33-41.

22. Hansson HA, Johansson B, Blomstrand C - Ultrastructural studies on cerebrovascular permeability in acute hypertension. Acta Neuropathol, 1975;32:187-198.

23. Hatashita S, Hoff JT, Ishii S - Focal brain edema associated with acute arterial hypertension. J Neurosurg, 1986;64:643-649.

24. Joas TA, Stevens WC - Comparison of the arrhythmic doses of epinefrine during forane, halothane, and fluroxene anesthesia in dogs. Anesthesiology, 1971;35:48-53.

25. Murr R, Berger $S$, Schürer $L$ et al - Influence of isoflurane, fentanyl, thiopental, and alpha-chloralose on formation of brain edema resulting from a focal cryogenic lesion. Anesth Analg, 1995;80:1108-1115.

26. Stullken EH, Sokoll MD - Intracranial pressure during hypotension and subsequent vasopressor therapy in anesthetized cats. Anesthesiology, 1975;42:425-431.

27. Rudehill A, Hjemdahl P, Sollevi A et al - Changes in cardiac metabolism, perfusion, ECG and plasma catecholamines during incresead intracranial pressure in the pig. Acta Anaesthesiol Scand, 1987;31:265-272.

28. Weinstein JD, Langfitt TW, Kassell NF - Vasopressor response to incresead intracranial pressure. Neurology, 1964;14: 1118-1131. 
29. Sullivan HG, Martinez J, Becker DP et al - Fluid-percussion model of mechanical brain injury in the cat. J Neurosurg, 1976;45:521-534.

30. Rosner MJ, Becker DP - Origin and evolution of plateau waves. Experimental observations and a teoretical model. J Neurosurg, 1984;60:312-324.

31. Fitch W, McDowall DG - Systemic vascular responses to increased intracranial pressure. 1. Effects of progressive epidural ballon expansion on intracranial pressure and systemic circulation. J Neurol Neurosurg Psychiatry, 1977;40:833-842.

32. Hariri RJ, Firlick AD, Shepard SR et al - Traumatic brain injury, hemorragic shock, and fluid resuscitation: effects on intracranial pressure and brain compliance. J Neurosurg, 1993;79:421-427.

33. Duke BJ, Ryu RK, Brega KE et al - Traumatic bilateral jugular vein thrombosis: case report and review of the literature. Neurosurgery, 1997;41:680-683.

34. Gennarelli TA, Thibault LE, Adams JH et al - Diffuse axonal injury and traumatic coma in the primate. Ann Neurol, 1982;12:564-574.

35, Drummond JC, Patel PM, Cole DJ et al - The effect of the reduction of colloid oncotic pressure, with and without reduction of osmolality, on post-traumatic cerebral edema. Anesthesiology. 1998;88:993-1002.

36. Siesjo BK - Cerebral circulation and metabolism. J Neurosurg, 1984;60:883-908.

37. Unterberg AW, Stroop R, Thomale EU et al - Characterisation of brain edema following "controlled cortical impact injury" in rats. Acta Neurochir, 1997;(Suppl70):106-108.

\section{RESUMEN}

Mizumoto N, Tango HK, Pagnocca ML - Efectos de la Hipertensión Arterial Inducida sobre la Complacencia y Presión de la Perfusión Encefálica en Hipertensión Intracraneana Experimental: Comparación entre Lesión Encefálica Criogénica y Balón Subdural

JUSTIFICATIVA Y OBJETIVOS: El trauma craneoencefálico (TCE) puede elevar la presión intracraneana $(P I C)$ y reducir la complacencia encefálica (CE). Diferentes lesiones son aplicadas en modelos de TCE que estudian las mismas variables. Como son usadas indistintamente, el objetivo es comparar la PIC y la CE en dos modelos de TCE.

MÉTODO: Dieciocho perros machos, anestesiados, ventilados y distribuidos eventualmente en dos grupos: BS - balón subdural $(n=9)$ y LC - lesión criogénica $(n=9)$. Análisis de la PIC, CE y presión de perfusión encefálica (PPE) en cinco momentos: final de la preparación (M0), encéfalo normal (M1), inicio de la lesión (M2), término de la lesión (M3) y lesión establecida (M4). CE es la variación de la PIC durante la hipertensión arterial inducida (HAl) en $50 \mathrm{mmHg}$ en M1 y M4. $P P E=$ presión arterial media (PAM) - PIC. Prueba t de Student emparejado para el mismo grupo en diferentes momentos y $t$ de Student para dos muestras diferentes para el mismo momento entre los grupos.

RESULTADOS: La PAM fue semejante en los grupos en los momentos estudiados ( $p=0,31$ en M0; $p=0,25$ en M1; $p=0,31$ en M2; $p=0,19$ en M3; $p=0,05$ en M4). La PIC fue semejante en los grupos en $M 0(p=0,27)$ y $M 1(p=0,21)$, pero diferente en $M 2$ $(p<0,001)$. La PIC se volvió semejante en los grupos en M3 ( $p=$ $0,39)$ y M4 $(p=0,98)$, se elevó en BS en $M 1(p=0,04)$ y $M 2(p=$ $0,01)$, pero no en M3 $(p=0,36)$ ni en M4 $(p=0,12)$. En el LC la PIC aumentó en $M 1(p<0,01), M 3(p<0,001)$ y M4 $(p<0,001)$, pero no en M2 $(p=0,18)$. Hubo aumento de la PPE en M1 $(p<$ $0,001)$ y M4 $(p<0,001)$, semejante en los grupos $(p=0,16$ en M1 y $p=0,21$ en M4). En M2 hubo reducción de la PPE en los grupos $(p<0,001)$, más intensa en $L C(p<0,001)$. En M3 hubo aumento de la PPE en BS $(p=0,02)$ y reducción en el LC $(p=$ $0,01)$, lo que hizo la PPE semejante en los grupos $(p=0,43)$. En M4 hubo aumento de la PPE semejante en los grupos ( $p=$ $0,16)$.

CONCLUSIONES: El efecto de la HAI en el modelo de LC es comparable al observado en el modelo de BS. Ese tipo de lesión debe ser mejor estudiada para establecer precisión en la proporción entre la extensión de la lesión y la reducción de la CE, aparentemente, un proceso gradual y evolutivo cuyos límites aún no son totalmente conocidos. 